\title{
Uncertain Timestamps in Linear State Estimation
}

Clas Veibäck, Gustaf Hendeby and Fredrik Gustafsson

The self-archived postprint version of this journal article is available at Linköping University Institutional Repository (DiVA):

http:// urn.kb.se/resolve?urn=urn:nbn:se:liu:diva- 157642

N.B.: When citing this work, cite the original publication.

Veibäck, C., Hendeby, G., Gustafsson, F., (2019), Uncertain Timestamps in Linear State Estimation, IEEE Transactions on Aerospace and Electronic Systems, 55(3), 1334-1346.

https:/ / doi.org/ 10.1109/TAES.2018.2869648

Original publication available at:

https:// doi.org/ 10.1109/TAES.2018.2869648

Copyright: Institute of Electrical and Electronics Engineers (IEEE)

http://www.ieee.org//

(C2019 IEEE. Personal use of this material is permitted. However, permission to reprint/republish this material for advertising or promotional purposes or for creating new collective works for resale or redistribution to servers or lists, or to reuse any copyrighted component of this work in other works must be obtained from the IEEE. 


\title{
Uncertain Timestamps in Linear State Estimation
}

\author{
Clas Veibäck, Gustaf Hendeby, Senior Member, IEEE, and Fredrik Gustafsson, Fellow, IEEE
}

\begin{abstract}
We consider a linear state estimation problem where, in addition to the usual timestamped measurements, observations with uncertain timestamps are available. Such observations could, e.g., come from traces left by a target in a tracking scenario or from witnesses of an event, and have the potential to improve the estimation accuracy significantly. We derive the posterior distribution and point estimators for a linear Gaussian smoothing formulation of this problem and illustrate with two numerical examples.
\end{abstract}

Index Terms-Uncertain Timestamps, Smoothing, Tracking, Linear State Estimation.

\section{INTRODUCTION}

A formulation of the linear state estimation problem that is useful in some scenarios is proposed with mixed measurements of two types:

M. Measurements with precise timestamps; and

o. Observations with uncertain timestamps.

Our interest in this problem originally comes from an idea for fusion of radar and droppings. In the project Smart Savannahs [1], we develop tracking algorithms for following rhinos inside a sanctuary. This can be done with sensors of type $\mathrm{M}$, complemented with manual observations of type $\mathrm{O}$ from the rangers. The rangers spend considerable time on patrol, recording indirect rhino sightings, including droppings, foot prints, resting places and browsing damages. They are all of type $\mathrm{O}$ and are particularly useful in the labelling problem, i.e., which track belongs to which rhino. Another application is in crime scene investigations, where the place of the crime is often known precisely, but not the time. For example, witness statements often contain uncertainty in both time and place, while surveillance cameras are precise in time. A third application is to estimate a bias in the type M measurements, when unbiased type $\mathrm{O}$ observations are available. The observations of type o need to be incorporated into a higher level data association algorithm, however, this paper will concern state estimation given a hypothesis of association.

The proposed approach is based on extending the optimal Bayes filter to consider uncertain stochastic timestamps, which usually are considered known exactly. Given a prior on the timestamps, the smoothed posterior distribution of the states conditioned on observations of both types, $\mathrm{M}$ and $\mathrm{O}$, is derived. This leads to a posterior that can be expressed as a compound distribution. This distribution is often difficult to compute analytically. However, for discrete timestamps and a linear Gaussian state-space model (LGSSM) the distribution can be computed exactly as a mixture of Gaussians, although the number of components may be prohibitively large. A sampling method is proposed to approximate the posterior in the general case and a few estimators are derived. The proposed sampling method computes the smoothing distribution for a large number of similar sets of timestamps. To improve the performance a method is derived to update an existing smoothing distribution in a single pass when inserting or removing an observation at an arbitrary point in time.

The problem considered in this paper bears a close resemblance to the problems of out-of-sequence and time-delayed measurements, in particular when the time-delay is uncertain. In such problems, the time delay is often assumed to be bounded, and mainly filtering applications are considered. The problem of time delays has been treated extensively over the years, however, relatively little work has concerned timing uncertainty. In the literature nothing has been found that extends the localized time delay to directly consider arbitrary timestamps with large uncertainty intervals, which is the topic of this paper. For this reason, a slightly different formulation of the problem is chosen.

The problem of fusing time-delayed measurements with a known timestamp can be solved using a Kalman filter with augmented states, first proposed in [2]. By augmenting the state vector with delayed state vectors, the covariance between a delayed measurement and the current state is implicitly computed, allowing for a correct measurement update. This naive approach is rather inefficient and various improved methods are proposed and discussed in [3]-[5]. The more complicated case when the measurements also might arrive out-of-sequence is treated in [6]-[9]. An efficient implementation is proposed in [10], which is also extended to handle delays in continuous time. The effects of time delays on the performance of systems are well studied, see e.g. [11]-[13]. Detections occurring at random, but known, times are considered in [14] and [15]. A reverse problem is considered in [16] where the time uncertainty is estimated for a position given the posterior.

Further, random sampling times and delays have been considered, see [17], as well as methods to estimate unknown static or slowly varying delays, see [18] and [19]. A few methods for managing discrete uncertain time delays in a filter are evaluated in [4]. The best performance is obtained by considering the problem as uncertain data association and using a probabilistic data association (PDA) filter [20], which is similar to the minimum mean squared error (MMSE) estimator derived in this paper. Worse performance is obtained using maximum likelihood association, which has similarities with the maximum a posteriori (MAP) estimator derived in this paper. However, the PDA filter is expensive, so instead they propose an approximate method using covariance union to combine the state posterior distributions when updated using the minimum and maximum time delay, respectively. This seems to perform well when localized time delays are considered, but the approximation is less reasonable when considering arbitrary timestamps. It is also not clear how to extend this method to deal with multiple uncertain observations with 
overlapping uncertainty intervals.

An uncertain time delay problem where the true timestamp is intermittently available is considered in [21]. However, a measurement of the timestamp is obtained for each sample with additive Gaussian noise. The parameters of the noise are jointly estimated with the state in a filter. The proposed filter method is to approximate the augmented state posterior using a Monte Carlo method that sequentially samples from the timestamp posterior distribution. This bears some resemblance with the sampling approach proposed in this paper, but the chosen sampling method requires that the order of the timestamps is known and does not directly extend to large or overlapping uncertainty intervals.

A modification of the probabilistic multiple hypothesis tracker (PMHT), where in addition to target data association uncertainty, uncertain time delays are considered, is proposed in [22]. The algorithm applies the expectationmaximization (EM) method [23] in a sliding window to obtain a filter estimate, initialized by the prediction of the estimate from the previous time step. A special case of this method is derived in this paper to efficiently compute the maximum a posteriori (MAP) estimate of the states, modified to consider arbitrary timestamps.

Preliminary results of this work considering only a single observation with an uncertain timestamp are already published in [24] and [25]. The previous work is extended in this paper to consider multiple observations with timestamps that are uncertain in continuous time, rather than discrete time, and to propose methods for managing the increased complexity.

\section{Continuous-Discrete Model}

The underlying model considered is a continuous-time dynamic model with discrete-time measurements of type $\mathrm{M}$. Although, some results are more general, the method is derived for a linear Gaussian state-space model.

\section{A. Linear Gaussian State-Space Model}

A linear Gaussian time-invariant continuous-discrete statespace model (LGSSM) is considered in this paper. The dynamical model is given by the stochastic differential equation [26]

$$
d \mathbf{x}(t)=\mathbf{A x}(t) d t+d \boldsymbol{\beta}(t),
$$

where the stochastic process $\mathbf{x}(t) \in \mathbb{R}^{n}$ is the state vector at time $t, \mathbf{A}$ is the system matrix, $\boldsymbol{\beta}(t)$ is a Brownian motion process with $\mathbf{E}\left[d \boldsymbol{\beta}(t) d \boldsymbol{\beta}^{T}(t)\right]=\mathbf{Q} d t$ and $\mathbf{Q}$ is a spectral density matrix. This is a special case of an Itô stochastic differential equation and the integrals of the noise for nonoverlapping intervals are assumed to be independent, so the process in (1) satisfies the Markov property. An independent prior on the state at time $t_{0}$ is given by $\mathbf{x}\left(t_{0}\right) \sim \mathcal{N}\left(\overline{\mathbf{x}}_{0}, \mathbf{P}_{0}\right)$, where $\mathcal{N}(\boldsymbol{\mu}, \boldsymbol{\Sigma})$ denotes a Gaussian distribution with mean $\boldsymbol{\mu}$ and covariance $\boldsymbol{\Sigma}$. The measurement model, with independent noise, is

$$
\mathbf{y}_{j}=\mathbf{H}_{j}^{y} \mathbf{x}\left(t_{j}^{y}\right)+\mathbf{e}_{j}^{y}, \quad j \in \mathcal{J},
$$

where $\mathbf{y}_{j} \in \mathbb{R}^{m_{y}}$ is a noisy measurement at time $t_{j}^{y}, \mathcal{J}=$ $\{1, \ldots, J\}, \mathbf{H}_{j}^{y}$ is the observation matrix and $\mathbf{e}_{j}^{y} \sim \mathcal{N}\left(\mathbf{0}, \mathbf{R}_{j}^{y}\right)$.
The LGSSM is a Gauss-Markov process [26], and the posterior distribution of the state $p(\mathbf{x}(\cdot) \mid \mathcal{Y})$ for this model can be computed analytically for any instants of time, where $\mathbf{x}(\cdot)$ denotes the entire state trajectory in continuous time and $\mathcal{Y}=\left\{\mathbf{y}_{j}\right\}_{j \in \mathcal{J}}$. Distributions are implicitly conditioned on the timestamps $\mathcal{T}_{y}=\left\{t_{j}^{y}\right\}_{j \in \mathcal{J}}$ where applicable. The continuoustime state trajectory can in many cases be marginalised to only consider the state $\mathbf{x}(t)$ at a single instant of time $t$ or the states $\mathcal{X}_{p}=\{\mathbf{x}(t)\}_{t \in \mathcal{T}_{p}}$ at multiple instants of time $\mathcal{T}_{p}$. In the sequel, sets of states will, when appropriate, be treated as column vectors with all states stacked.

\section{B. Discrete-Time Equivalent Model}

The differential equation in (1) can be solved analytically for a given instant of time by converting the model to a discretetime equivalent model [27]-[29]. The differential equation is discretized at time instants of interest as well as time instants for which there are measurements or a prior, given by the set $\mathcal{T}_{x}=\mathcal{T}_{p} \cup \mathcal{T}_{y} \cup t_{0}=\left\{t_{k}\right\}_{k \in 0 \cup \mathcal{K}}$, where $\mathcal{K}=\{1, \ldots, K\}$ and $t_{k-1}<t_{k}$. The equivalent stochastic difference equation is obtained by integrating (1) over the time interval $\left[t_{k-1}, t_{k}\right]$

$$
\mathbf{x}\left(t_{k}\right)=e^{\mathbf{A}\left(t_{k}-t_{k-1}\right)} \mathbf{x}\left(t_{k-1}\right)+\int_{t_{k-1}}^{t_{k}} e^{\mathbf{A}\left(t_{k}-\tau\right)} d \boldsymbol{\beta}(\tau),
$$

resulting in the discrete-time equivalent model

$$
\begin{array}{rlrl}
\mathbf{x}_{k} & =\mathbf{F}_{k, k-1} \mathbf{x}_{k-1}+\mathbf{w}_{k, k-1}, & & k \in \mathcal{K}, \\
\mathbf{y}_{j} & =\mathbf{H}_{j}^{y} \mathbf{x}\left(t_{j}^{y}\right)+\mathbf{e}_{j}^{y}, & j \in \mathcal{J}, \\
\mathbf{x}_{0} \sim \mathcal{N}\left(\overline{\mathbf{x}}_{0}, \mathbf{P}_{0}\right), &
\end{array}
$$

where $\mathbf{w}_{k, k-1} \sim \mathcal{N}\left(\mathbf{0}, \mathbf{Q}_{k, k-1}\right), \mathbf{x}_{k}=\mathbf{x}\left(t_{k}\right)$,

$$
\mathbf{F}_{i, j}=e^{\mathbf{A}\left(t_{i}-t_{j}\right)} \quad \text { and } \quad \mathbf{Q}_{i, j}=\int_{0}^{t_{i}-t_{j}} e^{\mathbf{A} \tau} \mathbf{Q} e^{\mathbf{A}^{T} \tau} d \tau .
$$

Full rank is assumed for $\mathbf{Q}_{i, j}$. Methods to compute the discretizations are described in [27]. Discretizations between $t_{k-1}$ and $t_{k}$ will sometimes be denoted by the subscript $k$ for convenience. The matrices in (5) satisfy, for all $t_{\tau} \in\left[t_{k-1}, t_{k}\right]$,

$$
\begin{aligned}
\mathbf{F}_{k, k-1} & =\mathbf{F}_{k, \tau} \mathbf{F}_{\tau, k-1}, \\
\mathbf{Q}_{k, k-1} & =\mathbf{F}_{k, \tau} \mathbf{Q}_{\tau, k-1} \mathbf{F}_{k, \tau}^{T}+\mathbf{Q}_{k, \tau}
\end{aligned}
$$

The joint posterior distribution for the states $\mathcal{X}=\left\{\mathbf{x}_{k}\right\}_{k \in \mathcal{K}}$ in the discrete-time model is given by $p(\mathcal{X} \mid \mathcal{Y})=\mathcal{N}(\mathcal{X} \mid \hat{\mathcal{X}}, \mathbf{P})$, which can be computed using Bayes' theorem [30]. The marginal distribution for each state can be computed efficiently using, e.g., the Rauch-Tung-Striebel smoother (RTSS) [31] or the Forward-Backward smoother [32].

\section{UNCERTAIN TIMESTAMPS}

A model incorporating observations with uncertain timestamps is proposed and its posterior distribution is derived. 


\section{A. Uncertain Timestamp Model}

The LGSSM in Section II-A is augmented by observations of type $\mathrm{O}$, resulting in the uncertain timestamp model (UTM). Let $\mathcal{Z}=\left\{\mathbf{z}_{i}\right\}_{i \in \mathcal{I}}$ be the set of observations and $\mathcal{T}=\left\{\tau_{i}\right\}_{i \in \mathcal{I}}$ represent their timestamps, where $\mathcal{I}=\{1, \ldots, I\}$. The observations $\mathbf{z}_{i} \in \mathbb{R}^{m_{z}}$ are modelled as independent conditioned on the states and the timestamps, and the model is given by

$$
\mathbf{z}_{i}=\mathbf{H}_{i}^{z} \mathbf{x}\left(\tau_{i}\right)+\mathbf{e}_{i}^{z}, \quad i \in \mathcal{I},
$$

where $\mathbf{H}_{i}^{z}$ is the observation matrix and $\mathbf{e}_{i}^{z} \sim \mathcal{N}\left(\mathbf{0}, \mathbf{R}_{i}^{z}\right)$ is noise. The timestamps are stochastic, but not necessarily independent, with a joint prior distribution modelled by

$$
\mathcal{T} \sim p(\mathcal{T})
$$

This distribution may, for example, be flat, given by context, obtained through measurements or estimated by the observer.

\section{B. Posterior Distribution of the Uncertain Timestamp Model}

The posterior distribution of the state in the UTM is computed by marginalization of the joint posterior distribution of the states and timestamps. It is given by the compound distribution

$$
p(\mathbf{x}(\cdot) \mid \mathcal{Y}, \mathcal{Z})=\int_{\mathcal{T}} p(\mathbf{x}(\cdot) \mid \mathcal{T}, \mathcal{Y}, \mathcal{Z}) \cdot p(\mathcal{T} \mid \mathcal{Y}, \mathcal{Z}) d \mathcal{T},
$$

where all possible timestamps $\mathcal{T}$ are integrated over. The first factor in the integrand is recognized as the posterior distribution of the state, conditioned on $\mathcal{T}$. This distribution can be computed using the various methods discussed in Sec. II-B, resulting in

$$
p(\mathbf{x}(\cdot) \mid \mathcal{T}, \mathcal{Y}, \mathcal{Z})=\mathcal{N}\left(\mathbf{x}(\cdot) \mid \hat{\mathbf{x}}^{\mathcal{T}}(\cdot), \mathbf{P}^{\mathcal{T}}(\cdot)\right),
$$

where the superscript indicates which timestamps are used. The second factor in the integrand in (8) is the posterior distribution of the uncertain timestamps $\mathcal{T}$, which can be computed up to a proportionality constant using Bayes' theorem and the independence assumption [30] as

$$
\begin{aligned}
w_{\mathcal{T}} & \triangleq p(\mathcal{T} \mid \mathcal{Y}, \mathcal{Z}) \propto p(\mathcal{Z} \mid \mathcal{T}, \mathcal{Y}) p(\mathcal{T}) \\
& =\prod_{i \in \mathcal{I}}\left[p\left(\mathbf{z}_{i} \mid \mathcal{T}_{1: i}, \mathcal{Y}, \mathcal{Z}_{1: i-1}\right) p\left(\tau_{i} \mid \mathcal{T}_{1: i-1}\right)\right]
\end{aligned}
$$

where the subscript notation $a: b=\{i\}_{i=a}^{b}$ is used to index a subset of the elements. The likelihood of observation $\mathbf{z}_{i}$ given a subset of the other observations and their timestamps is computed by marginalizing the joint posterior distribution of the observation $\mathbf{z}_{i}$ and the state $\mathbf{x}\left(\tau_{i}\right)$ at time $\tau_{i}$ as

$$
\begin{aligned}
p\left(\mathbf{z}_{i} \mid \mathcal{T}_{1: i}, \mathcal{Y}, \mathcal{Z}_{1: i-1}\right)=\int p\left(\mathbf{z}_{i}, \mathbf{x}\left(\tau_{i}\right) \mid \mathcal{T}_{1: i}, \mathcal{Y}, \mathcal{Z}_{1: i-1}\right) d \mathbf{x}\left(\tau_{i}\right) \\
\quad=\int p\left(\mathbf{z}_{i} \mid \mathbf{x}\left(\tau_{i}\right), \tau_{i}\right) \cdot p\left(\mathbf{x}\left(\tau_{i}\right) \mid \mathcal{Y}, \mathcal{Z}_{1: i-1}, \mathcal{T}_{1: i-1}\right) d \mathbf{x}\left(\tau_{i}\right) \\
\quad=\mathcal{N}\left(\mathbf{z}_{i} \mid \mathbf{H}_{i}^{z} \hat{\mathbf{x}}^{\mathcal{T}_{1: i-1}}\left(\tau_{i}\right), \mathbf{H}_{i}^{z} \mathbf{P}^{\mathcal{T}_{1: i-1}}\left(\tau_{i}\right)\left(\mathbf{H}_{i}^{z}\right)^{T}+\mathbf{R}_{i}^{z}\right) \\
\quad=\mathcal{N}\left(\mathbf{z}_{i} \mid \hat{\mathbf{z}}_{i}^{\mathcal{T}_{1: i-1}}\left(\tau_{i}\right), \mathbf{S}_{i}^{\mathcal{T}_{1: i-1}}\left(\tau_{i}\right)\right),
\end{aligned}
$$

where $\int \mathcal{N}(\mathbf{a} \mid, \mathbf{A b}, \mathbf{B}) \mathcal{N}(\mathbf{b} \mid \mathbf{c}, \mathbf{C}) d \mathbf{b}=\mathcal{N}\left(\mathbf{a} \mid \mathbf{A} \mathbf{c}, \mathbf{A C A}^{T}+\right.$ $\mathbf{B})$ is used in the third equality. The last equality defines a convenient notation for the mean and covariance of the prediction of $\mathbf{z}_{i}$ at time $\tau_{i}$ given the observations $\mathcal{Z}_{1: i-1}$, timestamps $\mathcal{T}_{1: i-1}$ and all the measurements $\mathcal{Y}$.

Computation of (10a) is performed stepwise. First the smoothed posterior $p(\mathbf{x}(\cdot) \mid \mathcal{Y})$ is computed for the measurements $\mathcal{Y}$. The likelihood of each observation is computed in turn at the given timestamp and the observation and timestamp are sequentially added as conditional variables to the posterior distribution. The order in which the observations are added is unimportant. The result is the product of all the computed likelihoods and the joint prior of the timestamps. The joint prior $p(\mathcal{T})$ can also be computed sequentially using the chain rule, which may be simpler in some cases. Inserting (9)-(10) into (8) gives the compound distribution

$$
p(\mathbf{x}(\cdot) \mid \mathcal{Y}, \mathcal{Z})=\int_{\mathcal{T}} w_{\mathcal{T}} \mathcal{N}\left(\mathbf{x}(\cdot) \mid \hat{\mathbf{x}}^{\mathcal{T}}(\cdot), \mathbf{P}^{\mathcal{T}}(\cdot)\right) d \mathcal{T},
$$

where $w_{\mathcal{T}}$ can be seen as the weight of the hypothesis of timestamps $\mathcal{T}$.

This class of distributions occurs in many fields of applications [33], [34], which has resulted in a number of established methods for computation. For a discrete timestamp prior, the posterior reduces to a Gaussian mixture distribution since $w_{\mathcal{T}}$ cannot have more support than $p(\mathcal{T})$. However, the posterior is difficult to compute analytically for more general priors. In such cases the posterior distribution needs to be approximated or computed numerically. The main challenges lie in obtaining a representative approximation of the timestamp posterior distribution in (10a) and obtaining a useful and informative presentation of the continuous-time state posterior distribution in (8).

\section{Point Estimators}

Point estimators [34] are used to provide useful and informative presentations of the posterior distributions in Sec. III-B. Three estimators are derived for the states and timestamps.

\section{A. Minimum Mean Squared Error State Estimator}

The MMSE estimator, derived in [34], for the state posterior distribution in (11) is the posterior mean given by

$$
\begin{gathered}
\hat{\mathbf{x}}^{\mathrm{MMSE}}(\cdot)=E_{\mathbf{x}(\cdot) \mid \mathcal{Y}, \mathcal{Z}}[\mathbf{x}(\cdot)]=E_{\mathcal{T} \mid \mathcal{Y}, \mathcal{Z}}\left[E_{\mathbf{x}(\cdot) \mid \mathcal{T}, \mathcal{Y}, \mathcal{Z}}[\mathbf{x}(\cdot)]\right] \\
=\int_{\mathcal{T}} w_{\mathcal{T}} E_{\mathbf{x}(\cdot) \mid \mathcal{T}, \mathcal{Y}, \mathcal{Z}}[\mathbf{x}(\cdot)] d \mathcal{T}=\int_{\mathcal{T}} w_{\mathcal{T}} \hat{\mathbf{x}}^{\mathcal{T}}(\cdot) d \mathcal{T} .
\end{gathered}
$$

For a continuous timestamp prior, the estimator generally needs to be approximated. The uncertainty of the estimator is approximated by the posterior covariance as

$$
\mathbf{P}^{\mathrm{MMSE}}(\cdot) \approx \int_{\mathcal{T}} w_{\mathcal{T}}\left(\mathbf{P}^{\mathcal{T}}(\cdot)+\tilde{\mathbf{x}}^{\mathcal{T}}(\cdot)\left(\tilde{\mathbf{x}}^{\mathcal{T}}(\cdot)\right)^{T}\right) d \mathcal{T},
$$

where $\tilde{\mathbf{x}}^{\mathcal{T}}(\cdot)=\hat{\mathbf{x}}^{\mathcal{T}}(\cdot)-\hat{\mathbf{x}}^{\mathrm{MMSE}}(\cdot)$.

\section{B. Maximum A Posteriori State Estimator}

The MAP estimator, derived in [34], for the state posterior distribution in (11) is given by

$$
\begin{aligned}
\hat{\mathbf{x}}^{\mathrm{MAP}}(\cdot) & =\underset{\mathbf{x}(\cdot)}{\arg \max } p(\mathbf{x}(\cdot) \mid \mathcal{Y}, \mathcal{Z}) \\
& =\underset{\mathbf{x}(\cdot)}{\arg \max } \int_{\mathcal{T}} w_{\mathcal{T}} \mathcal{N}\left(\mathbf{x}(\cdot) \mid \hat{\mathbf{x}}^{\mathcal{T}}(\cdot), \mathbf{P}^{\mathcal{T}}(\cdot)\right) d \mathcal{T} .
\end{aligned}
$$


Unlike the MMSE estimate, the MAP estimate for one instant of time also depends on the other time instants included in the maximization [34]. The MAP estimate at a particular instant of time will therefore differ depending on whether it is computed only for the state $\mathrm{x}(t)$ at that instant, the states $\mathcal{X}$ including other instants or the continuous-time trajectory $\mathbf{x}(\cdot)$. The estimator will in general, even in the case of a discrete timestamp prior, be impossible to compute analytically, so approximate or numerical methods are required.

\section{Maximum A Posteriori Timestamp Estimator}

The MAP estimator [34] for the posterior distribution of the timestamps in (10) is given by

$$
\begin{aligned}
\hat{\mathcal{T}}^{\mathrm{MAP}}=\underset{\mathcal{T}}{\arg \max } p(\mathcal{T} \mid \mathcal{Y}, \mathcal{Z})=\underset{\mathcal{T}}{\arg \max } \ln p(\mathcal{T} \mid \mathcal{Y}, \mathcal{Z})= \\
=\underset{\mathcal{T}}{\arg \max } \sum_{i \in \mathcal{I}} \ln p\left(\tau_{i} \mid \mathcal{T}_{1: i-1}\right)+ \\
\quad \underset{\mathcal{N}}{\ln \left(\mathbf{z}_{i} \mid \hat{\mathbf{z}}_{i}^{\mathcal{T}_{1: i-1}}\left(\tau_{i}\right), \mathbf{S}_{i}^{\mathcal{T}_{1: i-1}}\left(\tau_{i}\right)\right)} \\
=\underset{\mathcal{T}}{\arg \max } \sum_{i \in \mathcal{I}} 2 \ln p\left(\tau_{i} \mid \mathcal{T}_{1: i-1}\right)-\ln \left|\mathbf{S}_{i}^{\mathcal{T}_{1: i-1}}\left(\tau_{i}\right)\right| \\
\quad-\left\|\mathbf{z}_{i}-\hat{\mathbf{z}}_{i}^{\mathcal{T}_{1: i-1}}\left(\tau_{i}\right)\right\|_{\mathbf{S}_{i}^{\mathcal{T}_{1: i-1}\left(\tau_{i}\right)}}^{2}
\end{aligned}
$$

using the monotonically increasing property of logarithms. Numerical optimization [35] can be used to obtain an estimate.

\section{Approximate Computational Methods}

The timestamps of the UTM in (7) are assumed to be continuous in general. This results in difficulties in analytically optimizing and computing the integrals in (11)-(14). Three methods for approximately computing the posterior distributions and point estimators are presented.

\section{A. Discrete Timestamp Approximation}

One method is to approximate the prior on the timestamps in (7b) as a discrete distribution in the time instants $\mathcal{T}_{z}$. The posterior distribution then reduces from a compound distribution to a mixture distribution. This is effectively equivalent to replacing the integrals with summations in (11)-(13). With high enough resolution in time, the effect on the results is negligible, however, the choice is a trade-off between accuracy and computation time. For the UTM the resulting posterior is a mixture of Gaussians. The MMSE estimator for this distribution is straightforward to compute. However, the MAP estimator still requires numerical optimization methods [35]. This method was used in [24] when only a single observation of type $\mathrm{O}$ was considered. However, this option is not viable as the only approximation when multiple observations are considered since the number of components in the mixture grows exponentially with the number of type o observations.

\section{B. Gibbs Sampling}

Another method is to approximate the distribution in (11) by sampling. To compute the posterior distribution, one needs to integrate over an $I$-dimensional space. Even if the space is discretized, as discussed in the previous section, it is in general too costly to exhaustively explore the space of multiple observations when computing the distribution. As an alternative the distribution can be approximated using Gibbs sampling [36], [37]. The timestamps are sampled from the distribution $p(\mathcal{T} \mid \mathcal{Y}, \mathcal{Z})$ given in (10). The posterior distribution of the states conditional on the timestamps is computed using the methods discussed in Sec. II-B.

In the Gibbs sampler, samples of the timestamps are drawn iteratively conditional on the current samples of all other timestamps. The sample $\tau_{i}^{s}$ of the timestamp for observation $\mathbf{z}_{i}$ in iteration $s$ is drawn from the distribution

$$
\begin{aligned}
& p\left(\tau_{i} \mid \mathcal{T}_{\neg i}^{s}, \mathcal{Y}, \mathcal{Z}\right) \propto p\left(\mathbf{z}_{i} \mid \mathcal{Y}, \mathcal{Z}_{\neg i}, \mathcal{T}_{\neg i}^{s}, \tau_{i}\right) \cdot p\left(\tau_{i} \mid \mathcal{T}_{\neg i}^{s}\right) \\
& =\mathcal{N}\left(\mathbf{z}_{i} \mid \mathbf{H}_{i}^{z} \hat{\mathbf{x}}_{\neg i}^{\mathcal{T}_{\neg i}^{s}}\left(\tau_{i}\right), \mathbf{H}_{i}^{z} \mathbf{P}^{\mathcal{T}_{\neg i}^{s}}\left(\tau_{i}\right)\left(\mathbf{H}_{i}^{z}\right)^{T}+\mathbf{R}_{i}^{z}\right) \cdot p\left(\tau_{i} \mid \mathcal{T}_{\neg i}^{s}\right) \\
& =\mathcal{N}\left(\mathbf{z}_{i} \mid \hat{\mathbf{z}}_{i}^{\mathcal{T}_{\neg i}^{s}}\left(\tau_{i}\right), \mathbf{S}_{i}^{\mathcal{T}_{\neg i}^{s}}\left(\tau_{i}\right)\right) \cdot p\left(\tau_{i} \mid \mathcal{T}_{\neg i}^{s}\right),
\end{aligned}
$$

where $\mathcal{Z}_{\neg i}=\mathcal{Z} \backslash \mathbf{z}_{i}, \mathcal{T}_{\neg i}^{s}=\mathcal{T}_{1: i-1}^{s} \bigcup \mathcal{T}_{i+1: I}^{s-1}$ and similar notation as in (10b) is used in the last equality. In the case that the timestamps are discretized according to Sec. V-A, this distribution is simple to sample from, since it is gridded in one-dimension. However, for continuous timestamps rejection sampling [38], adaptive rejection Metropolis sampling [39], Metropolis sampling [40] or sampling/importance resampling [41], [42] can be used within the Gibbs sampler. A set $\left\{\mathcal{T}^{s}\right\}_{s=1}^{S}$ of timestamp samples is obtained to discretely approximate the distribution $p(\mathcal{T} \mid \mathcal{Y}, \mathcal{Z})$ in (10). The integral in (11) reduces to a sum over the samples and approximates the posterior distribution as

$$
p(\mathbf{x}(\cdot) \mid \mathcal{Y}, \mathcal{Z}) \approx \frac{1}{S} \sum_{s=1}^{S} \mathcal{N}\left(\mathbf{x}(\cdot) \mid \hat{\mathbf{x}}^{\mathcal{T}^{s}}(\cdot), \mathbf{P}^{\mathcal{T}^{s}}(\cdot)\right)
$$

The MMSE estimator presented in Sec. IV-A is similarly approximated using this discrete set of timestamps as

$$
\hat{\mathbf{x}}^{\operatorname{MMSE}}(\cdot) \approx \frac{1}{S} \sum_{s=1}^{S} \hat{\mathbf{x}}^{\mathcal{T}_{s}}(\cdot)
$$

\section{Expectation-Maximization of the State}

An approximate method that can be employed to compute the MAP of the states in (13) is a combination of the discrete timestamp approximation in Sec. V-A and the expectationmaximization (EM) method [23]. Although the method guarantees convergence, unfortunately there is no guarantee for convergence to the MAP estimate of the posterior distribution. This can be mitigated by using multiple starting points of the algorithm. A similar approach was presented in [22]. Interestingly, the method reduces to iteratively computing posterior distributions for LGSSMs.

The EM method iterates between computing an expectation over the timestamps and maximizing the expression with 
regards to the state. The expectation over the timestamps given the previous trajectory $\mathbf{x}^{-}(\cdot)$ is computed as

$$
\begin{aligned}
R & \left(\mathbf{x}(\cdot), \mathbf{x}^{-}(\cdot)\right) \\
= & \mathrm{E}_{\mathcal{T} \mid \mathcal{Z}, \mathcal{Y}, \mathbf{x}^{-}(\cdot)}[\ln (p(\mathcal{T}, \mathbf{x}(\cdot), \mathcal{Z}, \mathcal{Y}))] \\
= & \int p\left(\mathcal{T} \mid \mathcal{Z}, \mathbf{x}^{-}(\cdot)\right)[\ln p(\mathcal{Z} \mid \mathbf{x}(\cdot), \mathcal{T})+ \\
& \quad \ln p(\mathbf{x}(\cdot) \mid \mathcal{Y})+\ln p(\mathcal{T})+\ln p(\mathcal{Y})] d \mathcal{T} \\
= & C+\ln p(\mathbf{x}(\cdot) \mid \mathcal{Y})+ \\
& \sum_{i \in \mathcal{I}} \int w_{i}\left(\tau_{i}\right) \ln \mathcal{N}\left(\mathbf{z}_{i} \mid \mathbf{H}_{i}^{z} \mathbf{x}\left(\tau_{i}\right), \mathbf{R}_{i}^{z}\right) d \tau_{i}
\end{aligned}
$$

where $C$ is a constant independent of $\mathbf{x}(\cdot)$ and

$$
w_{i}\left(\tau_{i}\right)=\int p\left(\mathcal{T} \mid \mathcal{Z}, \mathbf{x}^{-}(\cdot)\right) d \mathcal{T}_{\neg i}=p\left(\tau_{i} \mid \mathcal{Z}, \mathbf{x}^{-}(\cdot)\right),
$$

where $\mathcal{T}_{\neg i}=\mathcal{T} \backslash \tau_{i}$. This is the marginal distribution of $\tau_{i}$ given all observations and the state. For independent priors on the timestamps the expression simplifies to

$$
w_{i}\left(\tau_{i}\right)=\frac{\mathcal{N}\left(\mathbf{z}_{i} \mid \mathbf{H}_{i}^{z} \mathbf{x}^{-}\left(\tau_{i}\right), \mathbf{R}_{i}^{z}\right) p\left(\tau_{i}\right)}{\int \mathcal{N}\left(\mathbf{z}_{i} \mid \mathbf{H}_{i}^{z} \mathbf{x}^{-}(\tau), \mathbf{R}_{i}^{z}\right) p\left(\tau_{i}=\tau\right) d \tau} .
$$

For dependent priors on the timestamps, the joint distribution in the integrand of (19) can be sampled, e.g. using a Gibbs sampler [36], and marginalized to obtain samples from $w_{i}\left(\tau_{i}\right)$. Using $\tilde{\mathbf{R}}_{i}^{z}(\tau)=w_{i}^{-1}(\tau) \mathbf{R}_{i}^{z}$ the sum in (18) reduces to

$$
\tilde{C}-\frac{1}{2} \int \sum_{i \in \mathcal{I}}\left\|\mathbf{z}_{i}-\mathbf{H}_{i}^{z} \mathbf{x}(\tau)\right\|_{\tilde{\mathbf{R}}_{i}^{z}(\tau)}^{2} d \tau .
$$

If the observation matrix is the same for all observations, such that $\mathbf{H}_{i}^{z}=\mathbf{H}^{z}$, the sum in (18) further reduces to

$$
\hat{C}-\frac{1}{2} \int\left\|\overline{\mathbf{z}}(\tau)-\mathbf{H}^{z} \mathbf{x}(\tau)\right\|_{\overline{\mathbf{R}}^{z}(\tau)}^{2} d \tau,
$$

where an equivalent observation is used, given by

$$
\begin{aligned}
\overline{\mathbf{R}}^{z}(\tau) & =\left[\sum_{i \in \mathcal{I}} w_{i}(\tau)\left(\mathbf{R}_{i}^{z}\right)^{-1}\right]^{-1} \\
\overline{\mathbf{z}}(\tau) & =\overline{\mathbf{R}}^{z}(\tau)\left[\sum_{i \in \mathcal{I}} w_{i}(\tau)\left(\mathbf{R}_{i}^{z}\right)^{-1} \mathbf{z}_{i}\right] .
\end{aligned}
$$

The trajectory maximizing (18) is obtained in the maximization step as

$$
\mathbf{x}^{+}(\cdot)=\underset{\mathbf{x}(\cdot)}{\arg \max } R\left(\mathbf{x}(\cdot), \mathbf{x}^{-}(\cdot)\right),
$$

where the + denotes the trajectory to use in the next iteration.

The expectation expression involves integrals over continuous time, which requires a continuous-time state trajectory to be estimated in each iteration. In practice, however, the state trajectory cannot be estimated in continuous time. One option for implementation is to discretize the prior [22] according to the discussion in Sec. V-A. This reduces the integrals in (18) and (20) to summations and allows the state to be considered in the discrete times $\mathcal{T}_{x}=t_{0} \cup \mathcal{T}_{p} \cup \mathcal{T}_{y} \cup \mathcal{T}_{z}$. Let $\mathcal{X}$ be a column vector of the states at the time instants in $\mathcal{T}_{x}$, then (18) becomes

$$
\begin{aligned}
& R\left(\mathcal{X}, \mathcal{X}^{-}\right) \\
& =\tilde{C}+\ln p(\mathcal{X} \mid \mathcal{Y})-\frac{1}{2} \sum_{i \in \mathcal{I}} \sum_{\tau_{i} \in \mathcal{T}_{z}}\left\|\mathbf{z}_{i}-\mathbf{H}_{i}^{z} \mathbf{x}\left(\tau_{i}\right)\right\|_{\tilde{\mathbf{R}}_{i}^{z}\left(\tau_{i}\right)}^{2} \\
& =\bar{C}-\frac{1}{2} \sum_{j \in \mathcal{J}}\left\|\mathbf{y}_{j}-\mathbf{H}_{j}^{y} \mathbf{x}\left(t_{j}^{y}\right)\right\|_{\tilde{\mathbf{R}}_{j}^{y}}^{2}-\frac{1}{2}\left\|\mathbf{x}\left(t_{0}\right)-\overline{\mathbf{x}}_{0}\right\|_{\mathbf{P}_{0}}^{2} \\
& -\frac{1}{2} \sum_{k \in \mathcal{K}}\left\|\mathbf{x}\left(t_{k}\right)-\mathbf{F}_{k} \mathbf{x}\left(t_{k-1}\right)\right\|_{\mathbf{Q}_{k}}^{2} \\
& -\frac{1}{2} \sum_{\tau \in \mathcal{T}_{z}} \sum_{i \in \mathcal{I}}\left\|\mathbf{z}_{i}-\mathbf{H}_{i}^{z} \mathbf{x}(\tau)\right\|_{\tilde{\mathbf{R}}_{i}^{z}(\tau)}^{2} .
\end{aligned}
$$

The trajectory $\mathcal{X}^{+}$maximizing this expression can be obtained as discussed in Sec. II-B, noting that this problem can be reformulated as an equivalent LGSSM by replacing the observations in the UTM with the pseudo-observations

$$
\mathbf{z}_{i}=\mathbf{H}_{i}^{z} \mathbf{x}(\tau)+\tilde{\mathbf{e}}_{i}^{z}(\tau), \quad i \in \mathcal{I}, \tau \in \mathcal{T}_{z},
$$

where $\tilde{\mathbf{e}}_{i}^{z}(\tau) \sim \mathcal{N}\left(\mathbf{0}, \tilde{\mathbf{R}}_{i}^{z}(\tau)\right)$.

\section{Methods For EFFicient Computation}

The methods proposed in Sec. $\mathrm{V}$ require the posterior distribution to be computed for a large number of timestamps. Instead of recomputing the posterior each time, methods to update an existing distribution are proposed.

\section{A. Rauch-Tung-Striebel Smoother}

The RTSS [31] is an efficient method for recursively computing the smoothed posterior distribution of an LGSSM. However, to obtain the posterior distribution for the full state vector, the smoothed cross-covariances between consecutive states are required [43]. These can be computed in the backward recursion by adding [30]

$$
\begin{aligned}
\mathbf{P}_{k-1, k} & =\mathbf{P}_{k-1 \mid k-1} \mathbf{F}_{k}^{T}\left(\mathbf{F}_{k} \mathbf{P}_{k-1 \mid k-1} \mathbf{F}_{k}^{T}+\mathbf{Q}_{k}\right)^{-1} \mathbf{P}_{k} \\
& =\mathbf{P}_{k-1 \mid k-1} \mathbf{F}_{k}^{T} \mathbf{P}_{k \mid k-1}^{-1} \mathbf{P}_{k},
\end{aligned}
$$

where $\mathbf{P}_{k}, \mathbf{P}_{k \mid k}$, and $\mathbf{P}_{k \mid k-1}$ are the smoothed, filter, and prediction covariances for state $\mathbf{x}_{k}$, respectively.

\section{B. State Injection}

Given the smoothed posterior distribution of the states $\mathcal{X}$ at the time instants in $\mathcal{T}_{x}$, one wants to obtain the joint posterior distribution of the states $\mathcal{X}$ and the state $\mathbf{x}\left(t_{\tau}\right)$ for an arbitrary time $t_{\tau}$, where $t_{k-1}<t_{\tau}<t_{k}$. This is straightforward to compute using the RTSS for the new set of time instants with a large amount of redundancy. However, a more efficient method is proposed for the LGSSM using the results in Proposition 1. See illustration in Figure 1.

Proposition 1. Given the posterior distribution $p(\mathcal{X} \mid \mathcal{Y})=$ $\mathcal{N}(\mathcal{X} \mid \hat{\mathcal{X}}, \mathbf{P})$ for the model in (4), consider that the model is modified by substituting the equation

$$
\mathbf{x}_{k}=\mathbf{F}_{k, k-1} \mathbf{x}_{k-1}+\mathbf{w}_{k, k-1}
$$




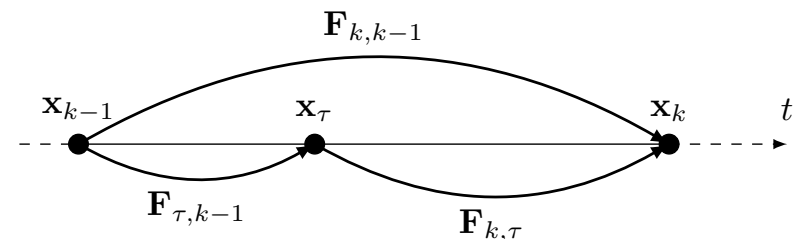

Fig. 1. Illustrates the injection of the state at time $t_{\tau}$ given the joint posterior distribution of the states at times $t_{k-1}$ and $t_{k}$. The modified equations need to satisfy the constraints in (6).

by the two equations

$$
\begin{aligned}
& \mathbf{x}_{\tau}=\mathbf{F}_{\tau, k-1} \mathbf{x}_{k-1}+\mathbf{w}_{\tau, k-1}, \\
& \mathbf{x}_{k}=\mathbf{F}_{k, \tau} \mathbf{x}_{\tau}+\mathbf{w}_{k, \tau},
\end{aligned}
$$

where $t_{k-1}<t_{\tau}<t_{k}$ and the transition and covariance matrices involved satisfy the conditions in (6).

The posterior distribution of the modified model is then given by

$$
\begin{aligned}
p\left(\left[\begin{array}{c}
\mathcal{X}_{m} \\
\mathbf{x}_{\tau}
\end{array}\right] \mid \mathcal{Y}\right)=\mathcal{N}( & {\left[\begin{array}{c}
\mathcal{X}_{m} \\
\mathbf{x}_{\tau}
\end{array}\right] \mid\left[\begin{array}{c}
\hat{\mathcal{X}} \\
\mathbf{K} \hat{\mathcal{X}}
\end{array}\right] } \\
& {\left.\left[\begin{array}{cc}
\mathbf{P} & \mathbf{P} \mathbf{K}^{T} \\
\mathbf{K P} & \mathbf{D}^{-1}+\mathbf{K P K}^{T}
\end{array}\right]\right), }
\end{aligned}
$$

where $\mathcal{X}_{m}$ denotes all states in the modified model excluding $\mathbf{x}_{\tau}$ and

$$
\begin{aligned}
\mathbf{I}_{k} \mathcal{X} & =\left(\begin{array}{c}
\mathbf{x}_{k-1} \\
\mathbf{x}_{k}
\end{array}\right), \\
\mathbf{D} & =\mathbf{Q}_{\tau, k-1}^{-1}+\mathbf{F}_{k, \tau}^{T} \mathbf{Q}_{k, \tau}^{-1} \mathbf{F}_{k, \tau}, \\
\mathbf{C} & =\left(\begin{array}{ll}
\mathbf{Q}_{\tau, k-1}^{-1} \mathbf{F}_{\tau, k-1} & \mathbf{F}_{k, \tau}^{T} \mathbf{Q}_{k, \tau}^{-1}
\end{array}\right), \\
\mathbf{K} & =\mathbf{D}^{-1} \mathbf{C I}_{k} .
\end{aligned}
$$

Proof. The proof is given in Appendix A.

Remark. The proposition implies that the states $\mathcal{X}$ are unaffected by the modification and that the only cross-covariances that are required are between consecutive states.

\section{Smoothed Posterior Distribution Update}

Given the smoothed posterior distribution of the states $\mathcal{X}$ at the time instants in $\mathcal{T}_{x}$, one wants to condition the distribution on an additional measurement $\mathbf{z}$ at a time $t_{\tau} \in \mathcal{T}_{x}$. Instead of recomputing the posterior distribution, a more efficient method is proposed for the LGSSM using the results in Proposition 2.

Proposition 2. Given the posterior distribution $p(\mathcal{X} \mid \mathcal{Y})=$ $\mathcal{N}(\mathcal{X} \mid \hat{\mathcal{X}}, \mathbf{P})$ for the model in (4), represented and uniquely determined by

$$
\begin{aligned}
\hat{\mathbf{x}}_{k} & =\mathrm{E}\left(\mathbf{x}\left(t_{k}\right)\right), & & k \in 0 \cup \mathcal{K}, \\
\mathbf{P}_{k} & =\operatorname{Cov}\left(\mathbf{x}\left(t_{k}\right)\right), & & k \in 0 \cup \mathcal{K}, \\
\mathbf{P}_{k-1, k} & =\operatorname{Cov}\left(\mathbf{x}\left(t_{k-1}\right), \mathbf{x}\left(t_{k}\right)\right), & & k \in \mathcal{K},
\end{aligned}
$$

consider that the model is modified by adding the equation

$$
\mathbf{z}=\mathbf{H x}\left(t_{\tau}\right)+\mathbf{e}, \quad \mathbf{e} \sim \mathcal{N}(\mathbf{0}, \mathbf{R}),
$$

where $\tau \in 0 \cup \mathcal{K}$. The posterior distribution $p(\mathcal{X} \mid \mathcal{Y}, \mathbf{z})=$ $\mathcal{N}\left(\mathcal{X} \mid \hat{\mathcal{X}}^{+}, \mathbf{P}^{+}\right)$of the modified model is then given by

$$
\begin{aligned}
\hat{\mathbf{x}}_{\tau}^{+} & =\hat{\mathbf{x}}_{\tau}+\mathbf{P}_{\tau} \mathbf{H}^{T} \mathbf{S}_{\tau}^{-1}\left(\mathbf{z}-\mathbf{H} \hat{\mathbf{x}}_{\tau}\right), \\
\mathbf{P}_{\tau}^{+} & =\mathbf{P}_{\tau}-\mathbf{P}_{\tau} \mathbf{H}^{T} \mathbf{S}_{\tau}^{-1} \mathbf{H} \mathbf{P}_{\tau},
\end{aligned}
$$

for the instant of time $t_{\tau}$, where $\mathbf{S}_{\tau}=\mathbf{H} \mathbf{P}_{\tau} \mathbf{H}^{T}+\mathbf{R}$, and recursively computed by

$$
\begin{aligned}
\hat{\mathbf{x}}_{k}^{+} & =\hat{\mathbf{x}}_{k}+\mathbf{K}_{k}^{b}\left(\hat{\mathbf{x}}_{k+1}^{+}-\hat{\mathbf{x}}_{k+1}\right), \\
\mathbf{P}_{k, k+1}^{+} & =\mathbf{P}_{k, k+1}+\mathbf{K}_{k}^{b}\left(\mathbf{P}_{k+1}^{+}-\mathbf{P}_{k+1}\right), \\
\mathbf{P}_{k}^{+} & =\mathbf{P}_{k}+\left(\mathbf{P}_{k, k+1}^{+}-\mathbf{P}_{k, k+1}\right)\left(\mathbf{K}_{k}^{b}\right)^{T},
\end{aligned}
$$

for $k<\tau$, where $\mathbf{K}_{k}^{b}=\mathbf{P}_{k, k+1} \mathbf{P}_{k+1}^{-1}$, and by

$$
\begin{aligned}
\hat{\mathbf{x}}_{k}^{+} & =\hat{\mathbf{x}}_{k}+\mathbf{K}_{k}^{f}\left(\hat{\mathbf{x}}_{k-1}^{+}-\hat{\mathbf{x}}_{k-1}\right), \\
\mathbf{P}_{k-1, k}^{+} & =\mathbf{P}_{k-1, k}+\left(\mathbf{P}_{k-1}^{+}-\mathbf{P}_{k-1}\right)\left(\mathbf{K}_{k}^{f}\right)^{T}, \\
\mathbf{P}_{k}^{+} & =\mathbf{P}_{k}+\mathbf{K}_{k}^{f}\left(\mathbf{P}_{k-1, k}^{+}-\mathbf{P}_{k-1, k}\right) .
\end{aligned}
$$

for $k>\tau$, where $\mathbf{K}_{k}^{f}=\mathbf{P}_{k-1, k}^{T} \mathbf{P}_{k-1}^{-1}$.

Proof. The proof is presented in Appendix B.

Combining (33)-(35) results in a method for efficiently updating a smoothed posterior distribution with an additional measurement, described in Algorithm 1 and previously published in [25]. A previously added measurement can be removed by subtracting its contribution, which artificially can be done using the algorithm together with a negative measurement noise covariance matrix. The main advantage of this approach is that only a single pass is required to update the smoothed posterior distribution compared to two passes required for the RTSS. In addition, the approach allows for further reduction in computation cost by terminating the forward and backward updates early when the contributions from the updates in (34) and (35) are negligible.

\section{ILLUSTRATIONS}

Two scenarios considering a single and multiple observations, respectively, are presented to illustrate and analyse the proposed methods.

\section{A. One-Dimensional Scenario with a Single Observation}

The purpose of this scenario is to illustrate the effects of the timestamp uncertainty on the posterior distribution in one dimension. This scenario was originally considered in [24]. A nearly constant position model is used on the form

$$
\begin{aligned}
d x(t) & =d \beta(t) & & E\left(d \beta^{2}(t)\right)=Q d t, \\
y_{1} & =x\left(t_{1}^{y}\right)+e_{1}^{y}, & & e_{1}^{y} \sim \mathcal{N}\left(0, R_{1}^{y}\right), \\
z_{1} & =x\left(\tau_{1}\right)+e_{1}^{z}, & & e_{1}^{z} \sim \mathcal{N}\left(0, R_{1}^{z}\right), \\
x(0) & \sim \mathcal{N}\left(\bar{x}_{0}, P_{0}\right) & &
\end{aligned}
$$

where the state $x(t)$ is the position, $\bar{x}_{0}=0 \mathrm{~km}, y_{1}=1 \mathrm{~km}$, $t_{1}^{y}=1 \mathrm{~h}$ and $z_{1}=0.5 \mathrm{~km}$. The specifications used are $Q=2.78 \cdot 10^{-3} \mathrm{~km}^{2} \mathrm{~h}^{-1}$ and $R_{1}^{y}=P_{0}=R_{1}^{z}=0.01 \mathrm{~km}^{2}$. The prior distribution of $\tau_{1}$ is uniformly distributed over the considered time interval between $0 \mathrm{~h}$ and $1 \mathrm{~h}$, which is relatively uninformative, and is discretized uniformly in 600 

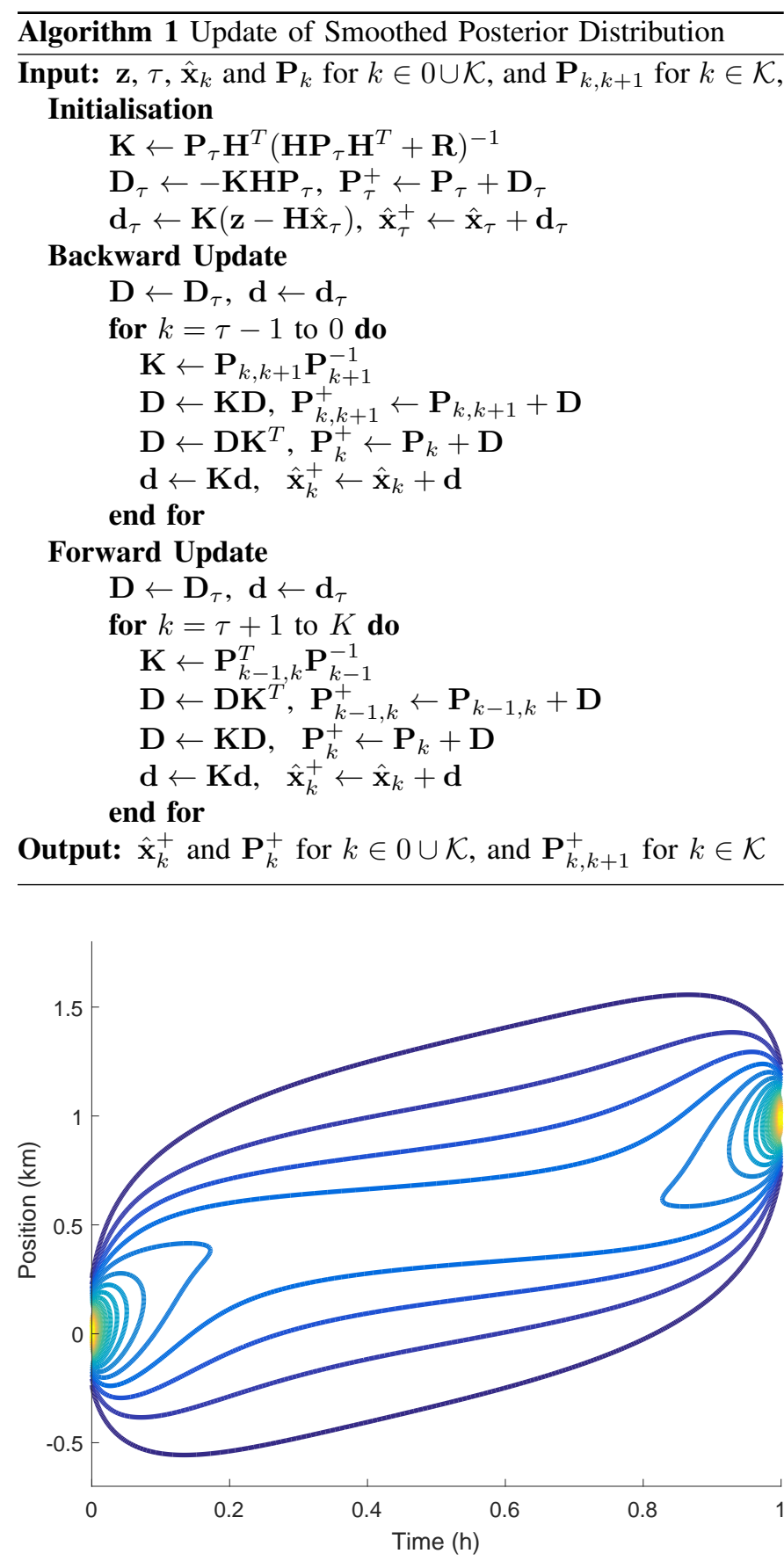

Fig. 2. Contour plot of the posterior distribution for the first scenario.

steps. Using (5), the discretized matrices are computed as $F_{k}=1$ and $Q_{k}=Q \cdot\left(t_{k}-t_{k-1}\right)$. With the discretization, the posterior distribution can be computed exactly and is shown in Fig. 2. Point estimators are shown in Fig. 3 together with the estimate for the original model given only the measurement. The prior and posterior distributions of the timestamp are shown in Fig. 4.

Assuming noise-free measurements and observations, and a continuous underlying system, the intermediate value theorem [44] for the one-dimensional model already gives the information that $x(t)=z_{1}$ for some $t \in[0,1] \mathrm{h}$, so at first glance the observation does not seem to add new information.

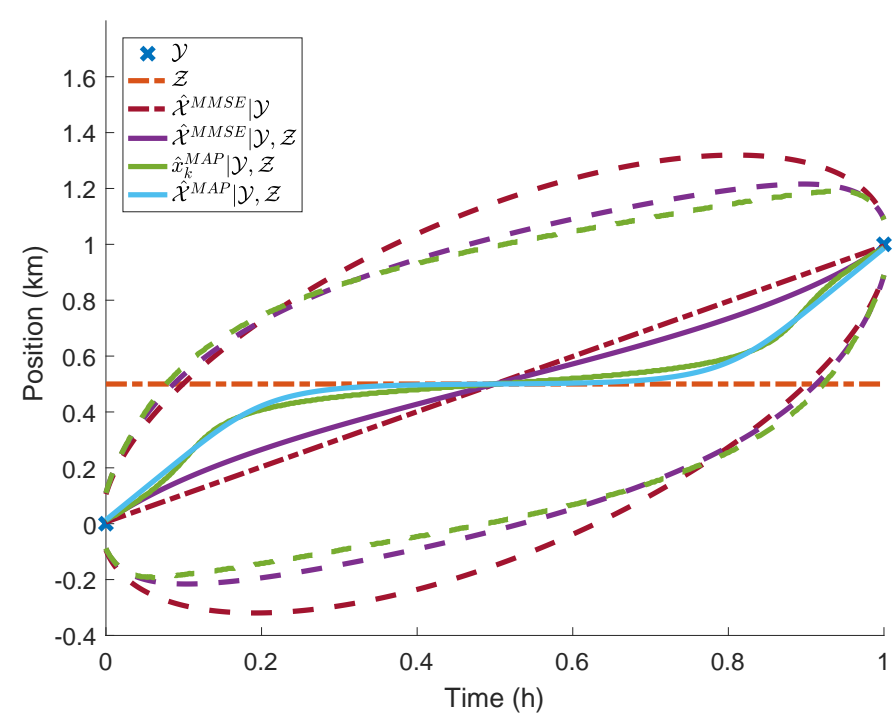

Fig. 3. The MMSE and MAP estimates and their uncertainties for the single observation scenario compared to the MMSE estimate given only the measurement.

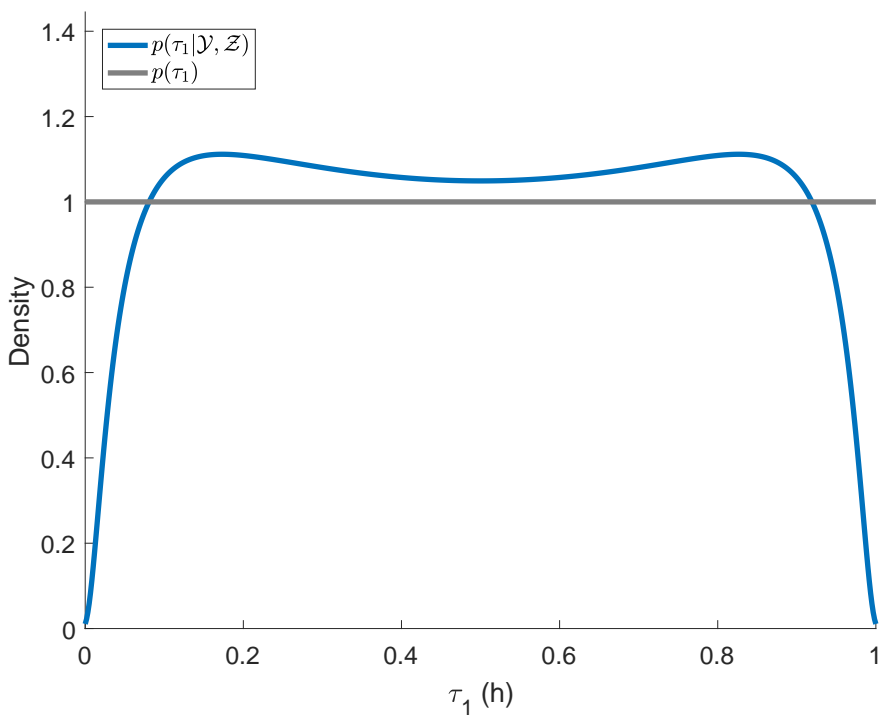

Fig. 4. The distributions $p\left(\tau_{1} \mid y_{1}, z_{1}\right)$ and $p\left(\tau_{1}\right)$ for the scenario with a single observation.

However, the state is more likely to be in the vicinity of the observation, explaining that the posterior is being pushed towards the observation in Fig. 2.

The MMSE estimator in Fig. 3 produces a trajectory that slightly tends towards the observation compared to the original trajectory given only the measurement. The reason for this behavior can be found in Fig. 4 where the posterior distribution of the timestamps is nearly uniform in the center. Given the timestamp, the trajectory passes near the observation at that time, attracting the compound distribution towards it and thus the MMSE estimate. Since additional information is available, the uncertainty of the estimate is seen to reduce significantly compared to the uncertainty in the original estimate.

This behavior is even more pronounced for the MAP estimators in Fig. 3, shown for both the joint and marginal posterior distributions of the states. For the MAP estimators of the 
marginal states, this can intuitively be understood by realizing that at each instant of time within the approximately uniform region of the timestamp posterior distribution in Fig. 4 the heights of the peaks will be approximately proportional to the inverse of the determinant of the covariance, and the smallest covariance is given by the Gaussian where the observation was used to update the posterior at the current time. This will attract the trajectory even more towards the observation than the MMSE estimate. The credible intervals of the posterior distributions for the marginal states also improves significantly over the original trajectory.

Inspecting Fig. 4 the dip in the middle of the posterior might seem unintuitive. However, the distribution is determined by (10b), and since the uncertainty is at its largest halfway between the end points, see Fig. 3, the dip can be understood.

\section{B. Two-Dimensional Scenario with Multiple Observations}

The purpose of this scenario is to demonstrate the effects of the timestamp uncertainty on the posterior distribution in two dimensions. A nearly constant velocity model is used on the form

$$
\begin{array}{rlrl}
d \mathbf{x}(t)=\mathbf{A} \mathbf{x}(t) d t+d \boldsymbol{\beta}(t), & E\left(d \boldsymbol{\beta}(t) d \boldsymbol{\beta}(t)^{T}\right)=\mathbf{Q} d t, \\
\mathbf{y}_{j} & =\mathbf{H x}\left(t_{j}^{y}\right)+\mathbf{e}_{j}^{y}, & & \mathbf{e}_{j}^{y} \sim \mathcal{N}\left(\mathbf{0}, \mathbf{R}^{y}\right), \\
\mathbf{z}_{i} & =\mathbf{H x}\left(\tau_{i}\right)+\mathbf{e}_{i}^{z}, & & \mathbf{e}_{i}^{z} \sim \mathcal{N}\left(\mathbf{0}, \mathbf{R}^{z}\right), \\
\mathbf{x}(0) & \sim \mathcal{N}\left(\overline{\mathbf{x}}_{0}, \mathbf{P}_{0}\right) & &
\end{array}
$$

where $\mathbf{y}_{j}$ with $\mathcal{T}_{y}=\{0: 6,12: 18,24: 30\} / 0.03 \mathrm{~s}$ for $j \in \mathcal{J}$ and $\mathbf{z}_{i}$ for $i \in \mathcal{I}=\{1,2\}$ are shown in Fig. 5. The state vector $\mathbf{x}(t)$ consists of the two-dimensional position and velocity, $\mathbf{A}=\left(\begin{array}{ll}0 & 1 \\ 0 & 0\end{array}\right) \otimes \mathbf{I}_{2}, \mathbf{H}=\left(\begin{array}{ll}1 & 0\end{array}\right) \otimes \mathbf{I}_{2}$ and $\mathbf{I}_{2}=\left(\begin{array}{ll}1 & 0 \\ 0 & 1\end{array}\right)$. Two priors for the timestamps are considered, one with independent timestamps distributed uniformly for $i \in \mathcal{I}$ over the time interval $[0,1000] \mathrm{s}$ and another with similar joint distribution, but with the additional dependency that the timestamps are ordered such that $i>j$ implies that $\tau_{i}>\tau_{j}$. The specifications used are $\mathbf{Q}=\left(\begin{array}{ll}0 & 0 \\ 0 & q\end{array}\right) \otimes \mathbf{I}_{2}, q=5 \cdot 10^{-6} \mathrm{~m}^{2} \mathrm{~s}^{-3}, \mathbf{R}^{z}=$ $5 \cdot 10^{-5} \cdot \mathbf{I}_{2} \mathrm{~m}^{2}, \mathbf{R}^{y}=5 \cdot 10^{-4} \cdot \mathbf{I}_{2} \mathrm{~m}^{2}, \overline{\mathbf{x}}_{0}=\left(0 \mathrm{~m}, 0 \mathrm{~ms}^{-1}\right)^{T}$ and $\mathbf{P}_{0}=\operatorname{diag}\left(10^{9} \cdot \mathbf{I}_{2} \mathrm{~m}^{2}, \mathbf{I}_{2} \mathrm{~m}^{2} \mathrm{~s}^{-2}\right)$. Using (5), the discretized matrices are computed as $\mathbf{F}_{i, j}=\left(\begin{array}{cc}1 & T_{i, j} \\ 0 & 1\end{array}\right) \otimes \mathbf{I}_{2}$ and $\mathbf{Q}_{i, j}=q\left(\begin{array}{cc}T_{i, j}^{3} / 3 & T_{i, j}^{2} / 2 \\ T_{i, j}^{2} / 2 & T_{i, j}\end{array}\right) \otimes \mathbf{I}_{2}$, where $T_{i, j}=t_{i}-t_{j}$.

The model in (37) is suitable for tracking where the control input is unknown, but to generate data that resembles a target following a desired trajectory a different approach is employed. The measurements $\mathcal{Y}$ are manually selected as a nonnoisy version of $\mathcal{Y}$ in Fig. 5. The posterior distribution for the model in (37) given only these measurements is computed. The timestamps are sampled from a distribution that is uniform in $[200,400] \mathrm{s}$ for $\tau_{1}$ and uniform in $[600,800] \mathrm{s}$ for $\tau_{2}$ to obtain one observation in each gap. A trajectory is sampled from the Gaussian process posterior distribution at the timestamps corresponding to $\mathcal{T}_{y}, \mathcal{T}$ and $\mathcal{T}_{p}$, where $\mathcal{T}_{p}$ is 200 equidistant samples in the interval $[0,1000] \mathrm{s}$. The measurements $\mathcal{Y}$ and observations $\mathcal{Z}$ are sampled from this realization according to the corresponding models in (37). One such realization is shown in Fig. 5.

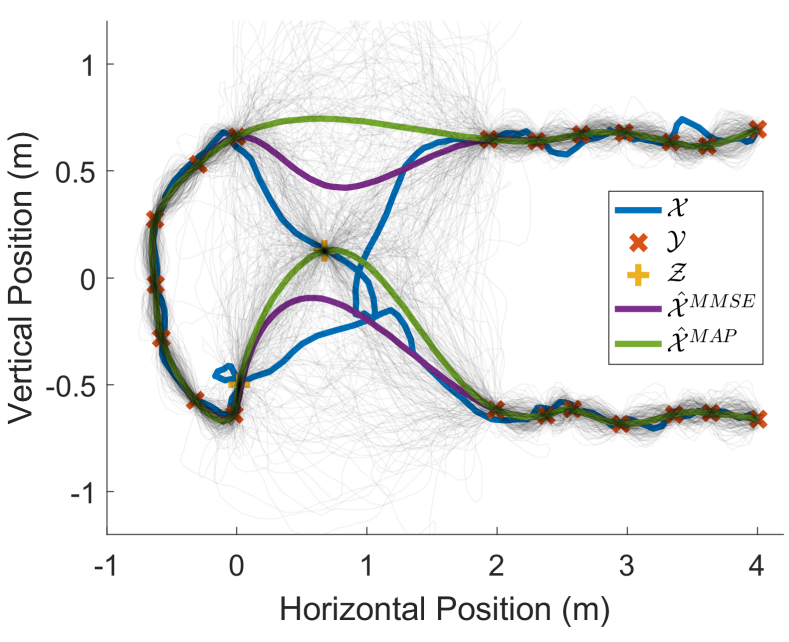

(a) Uniform prior.

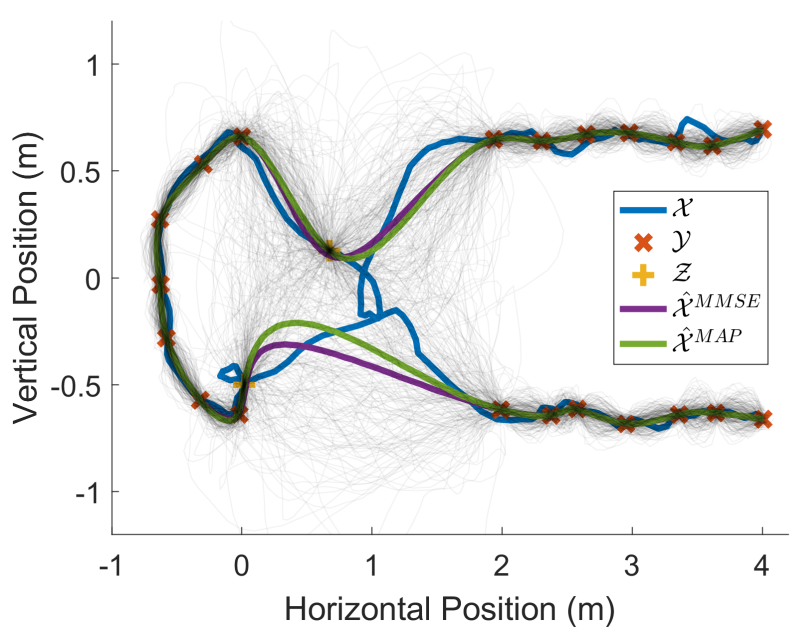

(b) Ordering prior.

Fig. 5. The realization and estimators for the two-dimensional scenario, with samples from the posterior showing in gray. The upper right measurement and topmost observation correspond to the first of their type.

Using sampling importance resampling within a Gibbs sampler, as described in Section V-B, with 100 and 200 samples respectively, the posterior distribution of the timestamps is sampled as shown in Fig. 6. For each sampled timestamp, a sample is drawn from the conditional posterior distribution to obtain samples from the posterior distribution of the states. The sampled states are shown in Fig. 5. The MMSE estimates of the distributions are shown as well as the MAP estimates. The latter is obtained by discretizing the prior in 200 points and applying the EM algorithm. For the dependent prior an additional Gibbs sampler with 300 samples was used inside the EM algorithm to approximate the timestamp distribution given a trajectory and the observations.

The sampled trajectories in Fig. 5 are rather dense near measurements, but more spread-out in the gaps where the uncertainty is large. One can see in Fig. 6a that with the uniform prior, the sampled timestamps form a few clusters at the peaks of the multi-modal posterior distribution. In Fig. $6 \mathrm{~b}$ one can see that infeasible timestamps are disregarded with 


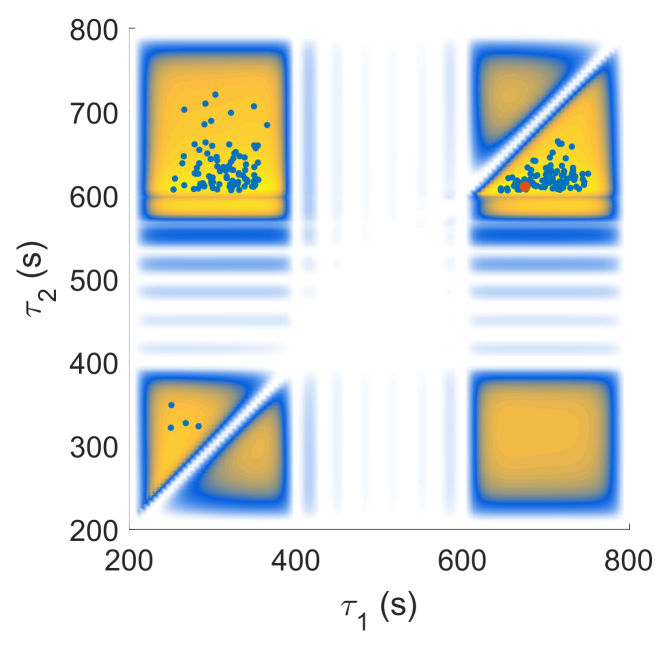

(a) Uniform prior.

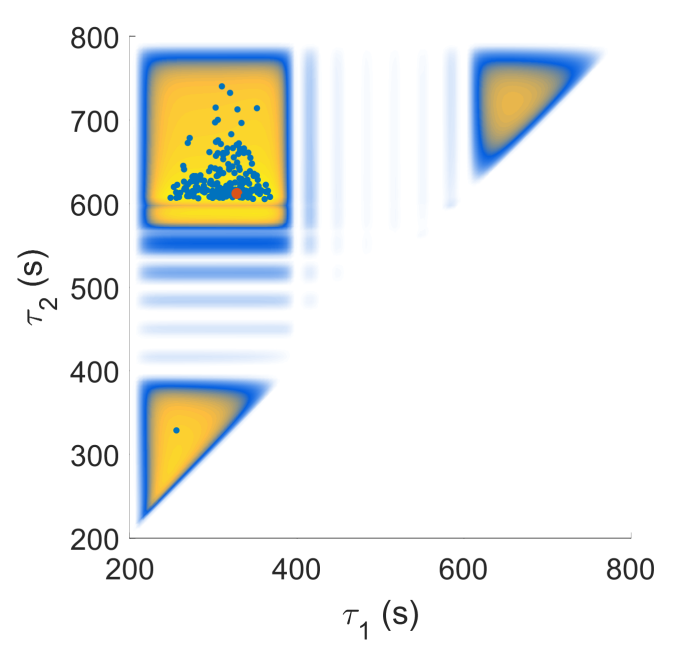

(b) Ordering prior.

Fig. 6. The posterior distribution of the timestamps for the two-dimensional scenario. White and blue indicate low probability and yellow indicates medium to high probability. Samples from the distribution are shown as blue dots and the MAP estimate is shown in red. The bottom right triangle in (b) has exactly zero probability due to the ordering prior.

the ordering prior, and almost all timestamps are sampled in one cluster around the highest peak.

An advantage of the MMSE estimator is that it is unbiased [34]. However for multimodal distributions a disadvantage is that the estimate might fall between modes, producing an unlikely estimate, as can be seen in Fig. 5a. It does not get close enough to the observations to be reasonable and tries to visit both observations both going out and coming back. The posterior distribution in Fig. 6b, however, is almost unimodal resulting in a MMSE estimate in Fig. 5b that is more feasible.

The MAP estimator will on the other hand produce likely estimates of the state, but at the cost of being biased [34]. The MAP estimators in Fig. 5 result in likely trajectories that visit the observations, although the estimated order might be incorrect as in Fig. 5a and 6a if the prior is not sufficiently informative. One disadvantage with the MAP estimator is that other likely trajectories corresponding to other modes in Fig. 6
TABLE I

RMSE OF POSITION AND VELOCITY AVERAGED OVER TIME AND 500 MONTE CARLO SIMULATIONS FOR VARIOUS ESTIMATORS.

\begin{tabular}{r|c|c}
\hline Estimator & Position $(\mathrm{m})$ & Velocity $(\mathrm{m} / \mathrm{s})$ \\
\hline Discarding $\mathcal{Z}$ & 0.2319 & 0.0066 \\
True $\mathcal{T}$ & 0.1562 & 0.0059 \\
\hline MMSE True prior & 0.1813 & 0.0061 \\
MAP True prior & 0.1931 & 0.0062 \\
JMAP True prior & 0.1932 & 0.0062 \\
\hline MMSE Ordering prior & 0.1863 & 0.0062 \\
MAP Ordering prior & 0.1939 & 0.0063 \\
JMAP Ordering prior & 0.2008 & 0.0063 \\
\hline MMSE Uniform prior & 0.1885 & 0.0062 \\
MAP Uniform prior & 0.2012 & 0.0063 \\
JMAP Uniform prior & 0.2064 & 0.0064
\end{tabular}

are completely disregarded. By using multiple initializations other modes may be obtained, which might be informative in presentation.

The bottom left and top right clusters in Fig. 6a represent that both observations are visited either on the way out or back, where the timestamp MAP estimate belongs to the latter. Note that the order of the timestamps for all samples in each cluster are the same. This is reasonable due to backtracking being unlikely. The top left cluster represents that the closest observation is visited on the way out and coming back, which represents the distribution that the timestamps were generated from. The infeasible timestamps using the ordering prior are rejected as expected in Fig. 6b, resulting in a timestamp MAP estimate that belongs to the correct cluster.

To compare the estimators the root mean square error (RMSE) averaged over the time instants in $\mathcal{T}_{p}$ and 500 Monte Carlo simulations is computed. The data is generated as previously described and the estimators use the model in (37). To obtain an upper and lower bound, the RMSE when discarding the observations $\mathcal{Z}$ and knowing the true timestamps $\mathcal{T}$ are computed. Three different priors are considered, the true prior used to generate the timestamps, the ordering prior and the uniform prior. For each prior the MMSE and MAP estimators are computed, as well as the joint MAP (JMAP) estimate of the states and timestamps. The latter is essentially obtained by numerically maximizing (14) and estimating the states using the resulting timestamps. The results for position and velocity estimates are presented in Table I.

For all three priors, the MMSE estimator outperforms the other estimators both for position and velocity. This is reasonable considering the purpose of the estimator is to minimize squared errors. The MAP estimator performs worse, but has the advantage of producing a likely estimate, which is not guaranteed for the MMSE estimator. The poor performance of the JMAP estimator can be explained by the lack of marginalization over the timestamps, which is inherent for the two other estimators. It is also seen that the performance improves with the amount of information put into the prior, but the improvement is relatively small compared to the bounds or to the difference between the estimators.

It should be noted that the particular scenario is designed to show the advantage of the additional observations. Nonetheless, it is shown that the performance improvement is not insignificant in such situations. 


\section{SUMmARY AND FUTURE WORK}

In this paper the problem of multiple observations with uncertain timestamps has been considered. Posterior distributions for the states and the uncertain timestamps have been derived as well as point estimators for a conditionally linear Gaussian state-space model. The exact solutions are often difficult to compute, so approximate methods have been proposed that can handle the complexity of the problem. The methods to use depend on many factors, such as the type and accuracy of the output required, the available computing power and the model. Regardless, the methods proposed in Sec. V will serve as useful components in a solution. In addition, exact methods for performing a measurement update in a smoothed posterior distribution, in one pass, as well as injecting an additional state into a smoothed posterior have been derived. These methods are useful in speeding up computations. The methods have been applied to two models, in one and two dimensions respectively, to attain an intuition for the effect of the uncertain timestamps. The performance of the method has been evaluated for the second model using Monte Carlo simulations and an improvement can be shown, especially for the MMSE estimator.

Several directions for future work are possible. The methods proposed in this paper could theoretically be used to compute the filtering distribution as well, but more efficient alternatives should be considered, e.g. filter banks, to approximate the filtering distribution in real time. Although the nature of the problem is nonlinear due to the uncertain timestamps, the proposed methods rely heavily on the underlying continuous model as well as the conditionally linear Gaussian structure of the model. Future work should extend the methods to consider a more general class of nonlinear models. An analysis of the possible benefits of using this model and under which circumstances the additional observations have most impact would also be interesting.

\section{ACKNOWLEDGEMENT}

This work has been supported by funding from Vinnova Industry Excellence Center LINK-SIC, the Swedish strategic research center Security Link and the Swedish Research Council through the project Scalable Kalman Filters.

\section{REFERENCES}

[1] (2017) Smart savannahs. [Online]. Available: http://wildlifesecurity.se/ smart-savannahs/

[2] S. Challa, R. J. Evans, and X. Wang, "A Bayesian solution and its approximations to out-of-sequence measurement problems," Information Fusion, vol. 4, no. 3, pp. 185-199, 2003.

[3] T. D. Larsen, N. A. Andersen, O. Ravn, and N. K. Poulsen, "Incorporation of time delayed measurements in a discrete-time Kalman filter," in Proceedings of the 37th IEEE Conference on Decision and Control, vol. 4, Dec 1998, pp. 3972-3977.

[4] S. J. Julier and J. K. Uhlmann, "Fusion of time delayed measurements with uncertain time delays," in Proceedings of the 2005 American Control Conference, June 2005, pp. 4028-4033.

[5] R. van der Merwe, E. A. Wan, and S. I. Julier, "Sigma-point Kalman filters for nonlinear estimation and sensor-fusion: Applications to integrated navigation," in Proceedings of the AIAA Guidance, Navigation \& Control Conference, 2004, pp. 16-19.

[6] R. D. Hilton, D. A. Martin, and W. D. Blair, "Tracking with timedelayed data in multisensor systems," NSWCDD, Dahlgren, VA, USA, Tech. Rep. TR-93/351, 1993.
[7] Y. Bar-Shalom, "Update with out-of-sequence measurements in tracking: exact solution," IEEE Transactions on Aerospace and Electronic Systems, vol. 38, no. 3, pp. 769-777, Jul 2002.

[8] M. Mallick, S. Coraluppi, and C. Carthel, "Advances in asynchronous and decentralized estimation," in 2001 IEEE Aerospace Conference Proceedings, vol. 4, 2001, pp. 4/1873-4/1888 vol.4.

[9] Y. Bar-Shalom, H. Chen, and M. Mallick, "One-step solution for the multistep out-of-sequence-measurement problem in tracking," IEEE Transactions on Aerospace and Electronic Systems, vol. 40, no. 1, pp. 27-37, Jan 2004.

[10] K. Zhang, X. R. Li, and Y. Zhu, "Optimal update with out-of-sequence measurements," IEEE Transactions on Signal Processing, vol. 53, no. 6, pp. 1992-2004, jun 2005

[11] J. Nilsson, B. Bernhardsson, and B. Wittenmark, "Stochastic analysis and control of real-time systems with random time delays," Automatica, vol. 34, no. 1, pp. 57-64, Jan. 1998.

[12] L. Schenato, "Optimal estimation in networked control systems subject to random delay and packet loss," in Proceedings of the 45th IEEE Conference on Decision and Control, Dec 2006, pp. 5615-5620.

[13] L. Shi, L. Xie, and R. M. Murray, "Kalman filtering over a packetdelaying network: A probabilistic approach," Automatica, vol. 45, no. 9 , pp. 2134-2140, 2009.

[14] Y. Bar-Shalom and G. Marcus, "Tracking with measurements of uncertain origin and random arrival times," IEEE Transactions on Automatic Control, vol. 25, no. 4, pp. 802-807, aug 1980.

[15] D. Snyder and P. Fishman, "How to track a swarm of fireflies by observing their flashes (corresp.)," IEEE Transactions on Information Theory, vol. 21, no. 6, pp. 692-695, Nov 1975.

[16] S. Coraluppi, C. Carthel, and A. Coon, "An MHT approach to multisensor passive sonar tracking," in Proceedings of the 21st International Conference on Information Fusion, Jul. 2018.

[17] S. C. Thomopoulos and L. Zhang, "Decentralized filtering with random sampling and delay," Information Sciences, vol. 81, no. 1, pp. 117-131, 1994.

[18] J. O. Nilsson, I. Skog, and P. Händel, "Joint state and measurement timedelay estimation of nonlinear state space systems," in 10th International Conference on Information Science, Signal Processing and their Applications (ISSPA 2010), May 2010, pp. 324-328.

[19] J. O. Nilsson and P. Händel, "Time synchronization and temporal ordering of asynchronous sensor measurements of a multi-sensor navigation system," in IEEE/ION Position, Location and Navigation Symposium, May 2010, pp. 897-902.

[20] S. S. Blackman and R. Popoli, Design and Analysis of Modern Tracking Systems. Artech House, 1999.

[21] M. Morelande, "Linear filtering with timing uncertainty," in Proceedings of the 11th International Conference on Information Fusion, Cologne, Germany, June 2008.

[22] B. Cheung, S. Davey, and D. Gray, "PMHT with timing uncertainty," IEEE Transactions on Aerospace and Electronic Systems, vol. 50, no. 4, pp. 2960-2973, October 2014.

[23] A. P. Dempster, N. M. Laird, and D. B. Rubin, "Maximum likelihood from incomplete data via the EM algorithm," Journal of the Royal Statistical Society. Series B (Methodological), vol. 39, no. 1, pp. 1-38, 1977.

[24] C. Veibäck, G. Hendeby, and F. Gustafsson, "On fusion of sensor measurements and observation with uncertain timestamp for target tracking," in Proceedings of the 19th International Conference on Information Fusion, Heidelberg, Germany, Jul. 2016, pp. 1268-1275.

[25] C. Veibäck, "Tracking of animals using airborne cameras," Licentiate's Thesis, Linköping University, Nov. 2016.

[26] A. Jazwinski, Stochastic Processes and Filtering Theory. New York, NY, USA: Academic Press, 1970, vol. 64.

[27] N. Wahlström, P. Axelsson, and F. Gustafsson, "Discretizing stochastic dynamical systems using Lyapunov equations," in Proceedings of the 19th World Congress of the International Federation of Automatic Control. International Federation of Automatic Control, 2014, pp. 3726-3731.

[28] Y. Bar-Shalom, T. Kirubarajan, and R. X. Li, Estimation with Applications to Tracking and Navigation. New York, NY, USA: John Wiley \& Sons, Inc., 2002

[29] A. Gelb, J. F. Kasper, R. A. Nash, C. F. Price, and A. A. Sutherland, Eds., Applied Optimal Estimation. Cambridge, MA, USA: MIT Press, 1974.

[30] S. Särkkä, Bayesian Filtering and Smoothing. Cambridge, United Kingdom: Cambridge University Press, 2013. 
[31] H. E. Rauch, F. Tung, and C. T. Striebel, "Maximum likelihood estimates of linear dynamic systems," AIAA Journal, vol. 3, no. 8, pp. 1445-1450, Aug. 1965.

[32] D. Fraser and J. Potter, "The optimum linear smoother as a combination of two optimum linear filters," IEEE Trans. Autom. Control, vol. 14, no. 4, pp. 387-390, Aug 1969.

[33] C. Röver and T. Friede, "Discrete approximation of a mixture distribution via restricted divergence," Journal of Computational and Graphical Statistics, vol. 26, no. 1, pp. 217-222, 2017.

[34] S. M. Kay, Fundamentals of Statistical Signal Processing: Estimation Theory. Upper Saddle River, NJ, USA: Prentice-Hall, Inc., 1993.

[35] J. Nocedal and S. J. Wright, Numerical Optimization, 2nd ed. New York, NY, USA: Springer, 2006.

[36] S. Geman and D. Geman, "Stochastic relaxation, Gibbs distributions, and the Bayesian restoration of images," IEEE Trans. on Pattern Analysis and Machine Intelligence, vol. 6, no. 6, pp. 721-741, Nov. 1984.

[37] A. E. Gelfand and A. F. M. Smith, "Sampling-based approaches to calculating marginal densities," Journal of the American Statistical Association, vol. 85, no. 410, pp. 398-409, 1990.

[38] A. Gelman, J. Carlin, H. Stern, D. Dunson, A. Vehtari, and D. Rubin, Bayesian Data Analysis, 3rd ed. Boca Raton, FL, USA: CRC Press, 2014.

[39] W. R. Gilks, N. G. Best, and K. K. C. Tan, "Adaptive rejection Metropolis sampling within Gibbs sampling," Journal of the Royal Statistical Society. Series C (Applied Statistics), vol. 44, no. 4, pp. 455472, 1995.

[40] N. Metropolis, A. Rosenbluth, M. Rosenbluth, A. Teller, and E. Teller, "Equation of state calculations by fast computing machines," The Journal of Chemical Physics, vol. 21, 1953.

[41] D. B. Rubin, "Using the SIR algorithm to simulate posterior distributions," in Bayesian Statistics 3, M. H. Bernardo, K. M. DeGroot, D. V. Lindley, and A. F. M. Smith, Eds. Oxford University Press, 1988

[42] A. F. M. Smith and A. E. Gelfand, "Bayesian statistics without tears: A sampling-resampling perspective," The American Statistician, vol. 46, no. 2, pp. 84-88, 1992.

[43] G. Meurant, "A review on the inverse of symmetric tridiagonal and block tridiagonal matrices," SIAM Journal on Matrix Analysis and Applications, vol. 13, no. 3, pp. 707-728, 1992.

[44] R. A. Adams, Calculus: A Complete Course, 5th ed. Pearson Education Canada, 2003.
Clas Veibäck is a Ph.D. student at the Division of Automatic Control, Department of Electrical Engineering, Linköping University, Sweden since 2013. In 2011, he received a M.Sc. degree in engineering physics from Uppsala University. The following years he worked with software development for unmanned aerial systems and a fighter aircraft. In November 2016 he received a Licentiate degree in Automatic Control. Clas's main research interest is in sensor fusion in general, with a focus on target tracking. Research projects have mainly involved tracking using cameras and radars as sensors, both stationary and as part of UAS platforms.

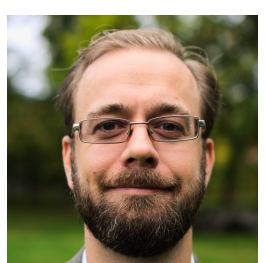

Gustaf Hendeby is an Associate Professor at the Division of Automatic Control, the Department of Electrical Engineering, Linköping University, Sweden. He received his M.Sc. in Applied Physics and Electrical Engineering in 2002 and his Ph.D. in Automatic Control in 2008, both from Linköping University. He worked as a Senior Researcher at the German Research Centre for Artificial Intelligence (DFKI) 2009-2011, and Senior Scientist at the Swedish Defence Research Agency (FOI) and held an adjunct Associate Professor position at Linköping University 2011-2015. Dr. Hendeby's main research interests are stochastic signal processing and sensor fusion with applications to nonlinear problems, target tracking, and simultaneous localization and mapping (SLAM). He has experience of both theoretical analysis as well as implementation aspects.

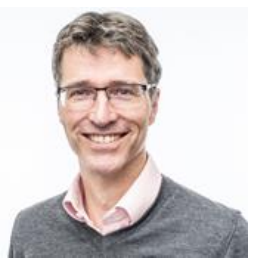

Fredrik Gustafsson is professor in Sensor Informatics at Department of Electrical Engineering, Linköping University, Sweden since 2005. He received his M.Sc. degree in electrical engineering 1988 and his Ph.D. degree in Automatic Control, 1992, both from Linköping University.

$\mathrm{He}$ is the author of five books, more than 200 conference papers, 100 journal papers and some 30 patents. His current h-index is 57 (Google Scholar). He has supervised $25 \mathrm{PhD}$ and more than 200 master theses.

He was an associate editor for IEEE Transactions of Signal Processing 2000-2006, IEEE Transactions on Aerospace and Electronic Systems 20102012, and EURASIP Journal on Applied Signal Processing 2007-2012. He was awarded the Arnberg prize by the Royal Swedish Academy of Science (KVA) 2004, elected member of the Royal Academy of Engineering Sciences (IVA) 2007, and elevated to IEEE Fellow 2011. In 2014, he was awarded a Distinguished Professor grant from the Swedish Research Council. He was an adjunct entrepreneurial professor at Twente University 2012-2013.

He was awarded the Harry Rowe Mimno Award 2011 for the tutorial "Particle Filter Theory and Practice with Positioning Applications", which was published in the AESS Magazine in July 2010. This paper was ranked \#4 out of 13077 articles published in the category IEEE Engineering Aerospace between 2010 and 2014 based on Web of Science citation data. He was a co-author of "Smoothed state estimates under abrupt changes using sum-ofnorms regularization" that received the Automatica paper prize in 2014.

$\mathrm{He}$ is a co-founder of the companies NIRA Dynamics (automotive safety, including tire pressure monitoring systems found in about 50 million cars today), Softube (plug-ins used in tens of thousands of music studios), and Senion (indoor navigation for smartphones deployed in more than 30 countries in all six continents). 


\title{
Uncertain Timestamps in Linear State Estimation - Supporting Document
}

\author{
Clas Veibäck, Gustaf Hendeby, Senior Member, IEEE, and Fredrik Gustafsson, Fellow, IEEE
}

\section{APPENDIX A \\ PROOF OF PROPOSITION 1}

This appendix presents the proof of Proposition 1. First, however, the posterior distribution for the LGSSM is derived to introduce useful notation.

The posterior distribution for the model in (4) can be expressed as

$$
\begin{aligned}
p(\mathcal{X} \mid \mathcal{Y}) \propto & p(\mathcal{Y} \mid \mathcal{X}) p(\mathcal{X}) \\
= & \prod_{j \in \mathcal{J}} p\left(\mathbf{y}_{j} \mid \mathbf{x}_{k_{j}}\right) \cdot \prod_{k \in \mathcal{K}} p\left(\mathbf{x}_{k} \mid \mathbf{x}_{k-1}\right) \cdot p\left(\mathbf{x}_{0}\right) \\
= & \prod_{j \in \mathcal{J}} \mathcal{N}\left(\mathbf{y}_{j} \mid \mathbf{H}_{j}^{y} \mathbf{x}_{k_{j}}, \mathbf{R}_{j}^{y}\right) \\
& \cdot \prod_{k \in \mathcal{K}} \mathcal{N}\left(\mathbf{x}_{k} \mid \mathbf{F}_{k} \mathbf{x}_{k-1}, \mathbf{Q}_{k}\right) \cdot \mathcal{N}\left(\mathbf{x}_{0} \mid \overline{\mathbf{x}}_{0}, \mathbf{P}_{0}\right),
\end{aligned}
$$

using Bayes' theorem and the independence and Markov assumptions. Rearranging each equation in (4) on the form

$$
\overline{\mathbf{y}}_{p}=\overline{\mathbf{H}}_{p} \mathcal{X}+\overline{\mathbf{E}}_{p}, \quad p \in \mathcal{P}=\{1, \ldots, K+J+1\},
$$

where $\operatorname{cov}\left(\overline{\mathbf{E}}_{p}\right)=\overline{\mathbf{R}}_{p}$, reduces (38) to

$$
\begin{aligned}
p(\mathcal{X} \mid \mathcal{Y})= & \prod_{p \in \mathcal{P}} \mathcal{N}\left(\overline{\mathbf{y}}_{p} \mid \overline{\mathbf{H}}_{p} \mathcal{X}, \overline{\mathbf{R}}_{p}\right) \\
& \propto \exp \left(-\frac{1}{2} \sum_{p \in \mathcal{P}}\left\|\overline{\mathbf{y}}_{p}-\overline{\mathbf{H}}_{p} \mathcal{X}\right\|_{\overline{\mathbf{R}}_{p}}^{2}\right) \\
& \propto \exp \left(-\frac{1}{2}\left\|\mathcal{X}-\mathcal{I}^{-1} \iota\right\|_{\mathcal{I}^{-1}}^{2}\right) \\
& \propto \mathcal{N}\left(\mathcal{X} \mid \mathcal{I}^{-1} \iota, \mathcal{I}^{-1}\right)=\mathcal{N}(\mathcal{X} \mid \hat{\mathcal{X}}, \mathbf{P}),
\end{aligned}
$$

using matrix completion of squares, where

$$
\begin{aligned}
\mathcal{I} & =\sum_{p \in \mathcal{P}} \overline{\mathbf{H}}_{p}^{T} \overline{\mathbf{R}}_{p}^{-1} \overline{\mathbf{H}}_{p}, \\
\iota & =\sum_{p \in \mathcal{P}} \overline{\mathbf{H}}_{p}^{T} \overline{\mathbf{R}}_{p}^{-1} \overline{\mathbf{y}}_{p} .
\end{aligned}
$$

The matrix $\mathcal{I}$ is often called the information matrix and $\iota$ the information state [1]. Note that each equation in the model in (4) contributes one term to the information matrix and one to the information state.

The posterior distribution in (29) for the modified model is computed similarly, where the following notation is used

$$
\begin{aligned}
p\left(\left[\begin{array}{c}
\mathcal{X}_{m} \\
\mathbf{x}_{\tau}
\end{array}\right] \mid \mathcal{Y}\right) & =\mathcal{N}\left(\left[\begin{array}{c}
\mathcal{X}_{m} \\
\mathbf{x}_{\tau}
\end{array}\right] \mid\left[\begin{array}{c}
\hat{\mathcal{X}}_{m} \\
\hat{\mathbf{x}}_{\tau}
\end{array}\right],\left[\begin{array}{cc}
\mathbf{P}_{m} & \mathbf{P}_{\tau m}^{T} \\
\mathbf{P}_{\tau m} & \mathbf{P}_{\tau}
\end{array}\right]\right) \\
& =\mathcal{N}\left(\mathcal{X} \mid \mathcal{I}_{m}^{-1} \iota_{m}, \mathcal{I}_{m}^{-1}\right) .
\end{aligned}
$$

Proof. The general approach of the proof is as follows. First, relationships between the information matrices and information states of the original and modified models are established. Second, partitioned inversion of the information matrix for the modified model is performed to show the equalities in (29).

In the remainder of the proof, the shorthands $\mathbf{F} \triangleq \mathbf{F}_{k, k-1}$, $\mathbf{F}_{1} \triangleq \mathbf{F}_{\tau, k-1}, \mathbf{F}_{2} \triangleq \mathbf{F}_{k, \tau}, \mathbf{Q} \triangleq \mathbf{Q}_{k, k-1}, \mathbf{Q}_{1} \triangleq \mathbf{Q}_{\tau, k-1}$ and $\mathbf{Q}_{2} \triangleq \mathbf{Q}_{k, \tau}$ are used, as well as the notations

$$
\mathbf{I}_{k, \tau} \mathcal{X}_{m}=\left(\begin{array}{c}
\mathbf{x}_{k-1} \\
\mathbf{x}_{k} \\
\mathbf{x}_{\tau}
\end{array}\right) \text {. }
$$

The information matrix and information state are augmented with an additional state, for which there exists no information, as

$$
\begin{aligned}
\mathcal{I}_{0} & =\left(\begin{array}{ll}
\mathcal{I} & \mathbf{0} \\
\mathbf{0} & \mathbf{0}
\end{array}\right)=\left(\begin{array}{cc}
\mathbf{P}^{-1} & \mathbf{0} \\
\mathbf{0} & \mathbf{0}
\end{array}\right), \\
\boldsymbol{\iota}_{0} & =\left(\begin{array}{l}
\boldsymbol{\iota} \\
\mathbf{0}
\end{array}\right)=\left(\begin{array}{c}
\mathbf{P}^{-1} \hat{\mathcal{X}} \\
\mathbf{0}
\end{array}\right) .
\end{aligned}
$$

With respect to information, this is in essence equivalent to the original model.

The removed equation in (28a) is rewritten on the form

$$
\begin{aligned}
\mathbf{0} & =\mathbf{H}_{-} \mathcal{X}_{m}+\mathbf{w}_{k, k-1}, \\
\mathbf{H}_{-} & =\left(\begin{array}{lll}
-\mathbf{F} & \mathbf{I} & \mathbf{0}
\end{array}\right) \mathbf{I}_{k, \tau} .
\end{aligned}
$$

The added equations in (28b) and (28c) are rewritten on the form

$$
\begin{aligned}
\mathbf{0} & =\mathbf{H}_{1} \mathcal{X}_{m}+\mathbf{w}_{\tau, k-1}, \\
\mathbf{H}_{1} & =\left(\begin{array}{lll}
-\mathbf{F}_{1} & \mathbf{0} & \mathbf{I}
\end{array}\right) \mathbf{I}_{k, \tau}
\end{aligned}
$$

and

$$
\begin{aligned}
\mathbf{0} & =\mathbf{H}_{2} \mathcal{X}_{m}+\mathbf{w}_{k, \tau}, \\
\mathbf{H}_{2} & =\left(\begin{array}{lll}
\mathbf{0} & \mathbf{I} & -\mathbf{F}_{2}
\end{array}\right) \mathbf{I}_{k, \tau} .
\end{aligned}
$$

Thus, the modification in (28) applied to (41a) and (41b) results in the following relationships between the information matrices and states of the original and modified models,

$$
\begin{aligned}
\mathcal{I}_{m}= & \mathcal{I}_{0}+ \\
& \mathbf{I}_{k, \tau}^{T}\left(-\mathbf{H}_{-}^{T} \mathbf{Q}^{-1} \mathbf{H}_{-}+\mathbf{H}_{1}^{T} \mathbf{Q}_{1}^{-1} \mathbf{H}_{1}+\mathbf{H}_{2}^{T} \mathbf{Q}_{2}^{-1} \mathbf{H}_{2}\right) \mathbf{I}_{k, \tau} \\
= & \mathcal{I}_{0}+\mathbf{I}_{k, \tau}^{T} \mathbf{H}_{m}^{T} \mathbf{R}_{m}^{-1} \mathbf{H}_{m} \mathbf{I}_{k, \tau}, \\
\boldsymbol{\iota}_{m}= & \iota_{0}+ \\
& \mathbf{I}_{k, \tau}^{T}\left(-\mathbf{H}_{-}^{T} \mathbf{Q}^{-1} \mathbf{0}+\mathbf{H}_{1}^{T} \mathbf{Q}_{1}^{-1} \mathbf{0}+\mathbf{H}_{2}^{T} \mathbf{Q}_{2}^{-1} \mathbf{0}\right)=\iota_{0},
\end{aligned}
$$


where

$$
\begin{aligned}
\mathbf{H}_{m} & =\left(\begin{array}{ccc}
-\mathbf{F} & \mathbf{I} & \mathbf{0} \\
-\mathbf{F}_{1} & \mathbf{0} & \mathbf{I} \\
\mathbf{0} & \mathbf{I} & -\mathbf{F}_{2}
\end{array}\right), \\
\mathbf{R}_{m} & =\left(\begin{array}{ccc}
-\mathbf{Q} & \mathbf{0} & \mathbf{0} \\
\mathbf{0} & \mathbf{Q}_{1} & \mathbf{0} \\
\mathbf{0} & \mathbf{0} & \mathbf{Q}_{2}
\end{array}\right)
\end{aligned}
$$

The middle part of the second term in (48a) is computed directly as

$$
\begin{aligned}
& \mathbf{H}_{m}^{T} \mathbf{R}_{m}^{-1} \mathbf{H}_{m}=\left(\begin{array}{cc}
\mathbf{A} & -\mathbf{C}^{T} \\
-\mathbf{C} & \mathbf{D}
\end{array}\right) \\
& =\left(\begin{array}{ccc}
-\mathbf{F}^{T} \mathbf{Q}^{-1} \mathbf{F}+\mathbf{F}_{1}^{T} \mathbf{Q}_{1}^{-1} \mathbf{F}_{1} & \mathbf{F}^{T} \mathbf{Q}^{-1} & -\mathbf{F}_{1}^{T} \mathbf{Q}_{1}^{-1} \\
\mathbf{Q}^{-1} \mathbf{F} & -\mathbf{Q}^{-1}+\mathbf{Q}_{2}^{-1} & -\mathbf{Q}_{2}^{-1} \mathbf{F}_{2} \\
-\mathbf{Q}_{1}^{-1} \mathbf{F}_{1} & -\mathbf{F}_{2}^{T} \mathbf{Q}_{2}^{-1} & \mathbf{Q}_{1}^{-1}+\mathbf{F}_{2}^{T} \mathbf{Q}_{2}^{-1} \mathbf{F}_{2}
\end{array}\right),
\end{aligned}
$$

where $\mathbf{C}$ and $\mathbf{D}$ correspond to (30c) and (30b). The resulting information matrix for the modified model in (48a) is then given by

$$
\begin{aligned}
\mathcal{I}_{m} & =\mathcal{I}_{0}+\mathbf{I}_{k, \tau}^{T} \mathbf{H}_{m}^{T} \mathbf{R}_{m}^{-1} \mathbf{H}_{m} \mathbf{I}_{k, \tau} \\
& =\left(\begin{array}{cc}
\mathcal{I}+\mathbf{I}_{k}^{T} \mathbf{A} \mathbf{I}_{k} & -\mathbf{I}_{k}^{T} \mathbf{C}^{T} \\
-\mathbf{C I} & \mathbf{D}
\end{array}\right)=\left(\begin{array}{cc}
\tilde{\mathbf{A}} & -\tilde{\mathbf{C}}^{T} \\
-\tilde{\mathbf{C}} & \tilde{\mathbf{D}}
\end{array}\right) .
\end{aligned}
$$

The covariance matrix in (29) is obtained using partitioned inversion [2] as

$$
\begin{aligned}
& {\left[\begin{array}{cc}
\mathbf{P}_{m} & \mathbf{P}_{\tau m}^{T} \\
\mathbf{P}_{\tau m} & \mathbf{P}_{\tau}
\end{array}\right]=\mathcal{I}_{m}^{-1}} \\
& =\left(\begin{array}{cc}
\mathbf{E}^{-1} & \mathbf{E}^{-1} \tilde{\mathbf{C}}^{T} \tilde{\mathbf{D}}^{-1} \\
\tilde{\mathbf{D}}^{-1} \tilde{\mathbf{C}} \mathbf{E}^{-1} & \tilde{\mathbf{D}}^{-1}+\tilde{\mathbf{D}}^{-1} \tilde{\mathbf{C}} \mathbf{E}^{-1} \tilde{\mathbf{C}}^{T} \tilde{\mathbf{D}}^{-1}
\end{array}\right),
\end{aligned}
$$

where

$$
\begin{aligned}
\mathbf{E} & =\tilde{\mathbf{A}}-\tilde{\mathbf{C}}^{T} \tilde{\mathbf{D}}^{-1} \tilde{\mathbf{C}}=\mathcal{I}+\mathbf{I}_{k}^{T} \mathbf{A} \mathbf{I}_{k}-\mathbf{I}_{k}^{T} \mathbf{C}^{T} \mathbf{D}^{-1} \mathbf{C} \mathbf{I}_{k} \\
& =\mathcal{I}+\mathbf{I}_{k}^{T}\left(\mathbf{A}-\mathbf{C}^{T} \mathbf{D}^{-1} \mathbf{C}\right) \mathbf{I}_{k} .
\end{aligned}
$$

The next step is to show that $\mathbf{A}=\mathbf{C}^{T} \mathbf{D}^{-1} \mathbf{C}$, which is achieved by showing that each of the four submatrices in $\mathbf{C}^{T} \mathbf{D}^{-1} \mathbf{C}$ are equivalent to the corresponding submatrices in $\mathbf{A}$ when the assumptions in (6) hold. The inverse of $\mathbf{D}$ is computed using the Woodbury formula [2] as

$$
\begin{aligned}
\mathbf{D}^{-1} & =\left(\mathbf{Q}_{1}^{-1}+\mathbf{F}_{2}^{T} \mathbf{Q}_{2}^{-1} \mathbf{F}_{2}\right)^{-1} \\
& =\mathbf{Q}_{1}-\mathbf{Q}_{1} \mathbf{F}_{2}^{T}\left(\mathbf{Q}_{2}+\mathbf{F}_{2} \mathbf{Q}_{1} \mathbf{F}_{2}^{T}\right)^{-1} \mathbf{F}_{2} \mathbf{Q}_{1} \\
& =\mathbf{Q}_{1}-\mathbf{Q}_{1} \mathbf{F}_{2}^{T} \mathbf{Q}^{-1} \mathbf{F}_{2} \mathbf{Q}_{1}
\end{aligned}
$$

The top left submatrix of $\mathbf{C}^{T} \mathbf{D}^{-1} \mathbf{C}$ is given by

$$
\begin{aligned}
&\left(-\mathbf{F}_{1}^{T} \mathbf{Q}_{1}^{-1}\right) \mathbf{D}^{-1}\left(-\mathbf{Q}_{1}^{-1} \mathbf{F}_{1}\right) \\
&=\mathbf{F}_{1}^{T} \mathbf{Q}_{1}^{-1}\left(\mathbf{Q}_{1}-\mathbf{Q}_{1} \mathbf{F}_{2}^{T} \mathbf{Q}^{-1} \mathbf{F}_{2} \mathbf{Q}_{1}\right) \mathbf{Q}_{1}^{-1} \mathbf{F}_{1} \\
&=\mathbf{F}_{1}^{T} \mathbf{Q}_{1}^{-1} \mathbf{F}_{1}-\mathbf{F}_{1}^{T} \mathbf{F}_{2}^{T} \mathbf{Q}^{-1} \mathbf{F}_{2} \mathbf{F}_{1} \\
&=\mathbf{F}_{1}^{T} \mathbf{Q}_{1}^{-1} \mathbf{F}_{1}-\mathbf{F}^{T} \mathbf{Q}^{-1} \mathbf{F} .
\end{aligned}
$$

The bottom left and transpose of top right submatrices are computed as

$$
\begin{aligned}
\left(-\mathbf{Q}_{2}^{-1} \mathbf{F}_{2}\right) \mathbf{D}^{-1}\left(-\mathbf{Q}_{1}^{-1} \mathbf{F}_{1}\right)= & \\
& \mathbf{Q}_{2}^{-1} \mathbf{F}_{2}\left(\mathbf{Q}_{1}-\mathbf{Q}_{1} \mathbf{F}_{2}^{T} \mathbf{Q}^{-1} \mathbf{F}_{2} \mathbf{Q}_{1}\right) \mathbf{Q}_{1}^{-1} \mathbf{F}_{1} \\
& =\mathbf{Q}_{2}^{-1}\left(\mathbf{I}-\mathbf{F}_{2} \mathbf{Q}_{1} \mathbf{F}_{2}^{T} \mathbf{Q}^{-1}\right) \mathbf{F} \\
& =\mathbf{Q}_{2}^{-1}\left(\mathbf{Q}_{2}+\mathbf{F}_{2} \mathbf{Q}_{1} \mathbf{F}_{2}^{T}-\mathbf{F}_{2} \mathbf{Q}_{1} \mathbf{F}_{2}^{T}\right) \mathbf{Q}^{-1} \mathbf{F} \\
& =\mathbf{Q}^{-1} \mathbf{F}
\end{aligned}
$$

The bottom right submatrix is computed, using the Woodbury formula backwards, as

$$
\begin{aligned}
\mathbf{Q}_{2}^{-1} & \mathbf{F}_{2} \mathbf{D}^{-1} \mathbf{Q}_{2}^{-1} \mathbf{F}_{2}= \\
& \mathbf{Q}_{2}^{-1} \mathbf{F}_{2}\left(\mathbf{Q}_{1}^{-1}+\mathbf{F}_{2}^{T} \mathbf{Q}_{2}^{-1} \mathbf{F}_{2}\right)^{-1} \mathbf{Q}_{2}^{-1} \mathbf{F}_{2} \\
& =\mathbf{Q}_{2}^{-1} \mathbf{F}_{2}\left(\mathbf{Q}_{1}^{-1}+\mathbf{F}_{2}^{T} \mathbf{Q}_{2}^{-1} \mathbf{F}_{2}\right)^{-1} \mathbf{Q}_{2}^{-1} \mathbf{F}_{2} \\
& +\mathbf{Q}_{2}^{-1}-\mathbf{Q}_{2}^{-1}=\mathbf{Q}_{2}^{-1}-\left(\mathbf{Q}_{2}+\mathbf{F}_{2}^{T} \mathbf{Q}_{1} \mathbf{F}_{2}\right)^{-1} \\
& =\mathbf{Q}_{2}^{-1}-\mathbf{Q}^{-1} .
\end{aligned}
$$

Using the above results,

$$
\begin{aligned}
& \mathbf{C}^{T} \mathbf{D}^{-1} \mathbf{C} \\
& =\left(\begin{array}{cc}
-\mathbf{F}^{T} \mathbf{Q}^{-1} \mathbf{F}+\mathbf{F}_{1}^{T} \mathbf{Q}_{1}^{-1} \mathbf{F}_{1} & \mathbf{F}^{T} \mathbf{Q}^{-1} \\
\mathbf{Q}^{-1} \mathbf{F} & -\mathbf{Q}^{-1}+\mathbf{Q}_{2}^{-1}
\end{array}\right)=\mathbf{A} .
\end{aligned}
$$

which reduces (54) to

$$
\mathbf{E}=\mathcal{I}=\mathbf{P}^{-1}=\mathbf{P}_{m}^{-1} .
$$

The cross-covariance $\mathbf{P}_{\tau m}$ in (53) reduces to

$$
\mathbf{P}_{\tau m}=\tilde{\mathbf{D}}^{-1} \tilde{\mathbf{C}} \mathbf{E}^{-1}=\mathbf{D}^{-1} \mathbf{C} \mathbf{I}_{k} \mathbf{P}=\mathbf{K P},
$$

and the covariance $\mathbf{P}_{\tau}$ in (53) reduces to

$$
\begin{aligned}
\mathbf{P}_{\tau} & =\tilde{\mathbf{D}}^{-1}+\tilde{\mathbf{D}}^{-1} \tilde{\mathbf{C}} \mathbf{E}^{-1} \tilde{\mathbf{C}}^{T} \tilde{\mathbf{D}}^{-1} \\
& =\mathbf{D}^{-1}+\mathbf{D}^{-1} \mathbf{C I}_{k} \mathbf{E}^{-1} \mathbf{I}_{k}^{T} \mathbf{C}^{T} \mathbf{D}^{-1} \\
& =\mathbf{D}^{-1}+\mathbf{K} \mathbf{P} \mathbf{K}^{T},
\end{aligned}
$$

using (52), (60) and (30d). This proves the covariance relation in (29).

The expectation in (29) reduces to

$$
\begin{aligned}
{\left[\begin{array}{c}
\hat{\mathcal{X}}_{m} \\
\hat{\mathbf{x}}_{\tau}
\end{array}\right] } & =\mathcal{I}_{m}^{-1} \iota_{m}=\mathcal{I}_{m}^{-1} \iota_{0}=\left(\begin{array}{c}
\mathbf{E}^{-1} \iota \\
\tilde{\mathbf{D}}^{-1} \tilde{\mathbf{C}} \mathbf{E}^{-1} \iota
\end{array}\right) \\
& =\left(\begin{array}{c}
\mathcal{I}^{-1} \iota \\
\mathbf{D}^{-1} \mathbf{C I}_{k} \mathcal{I}^{-1} \iota
\end{array}\right)=\left(\begin{array}{c}
\hat{\mathcal{X}} \\
\mathbf{K} \hat{\mathcal{X}}
\end{array}\right),
\end{aligned}
$$

using (40), (48b), (44b), (53), (60) and (61). This proves the expectation relation in (29) and concludes the proof.

To avoid inversion of $\mathbf{Q}_{1}$ and $\mathbf{Q}_{2}$, which might cause numerical instability for $t_{\tau}$ near $t_{k}$ or $t_{k-1}$, let $\mathbf{G}=\mathbf{Q}_{1} \mathbf{F}_{2}^{T} \mathbf{Q}^{-1}$ and rewrite

$$
\begin{aligned}
\mathbf{D}^{-1} & =\mathbf{Q}_{1}-\mathbf{Q}_{1} \mathbf{F}_{2}^{T} \mathbf{Q}^{-1} \mathbf{F}_{2} \mathbf{Q}_{1}=\left(\mathbf{I}-\mathbf{G F}_{2}\right) \mathbf{Q}_{1}, \\
\mathbf{K} & =\mathbf{D}^{-1} \mathbf{C}=\left(\left(\mathbf{I}-\mathbf{G F}_{2}\right) \mathbf{F}_{1} \quad \mathbf{G}\right),
\end{aligned}
$$

where $\mathbf{D}^{-1}$ and the left submatrix of $\mathbf{K}$ follow directly. The right submatrix assumes that $\mathbf{F}_{2}$ is invertible and is derived as $\mathrm{t}$

$$
\begin{aligned}
\mathbf{D}^{-1} \mathbf{F}_{2}^{T} \mathbf{Q}_{2}^{-1} & =\left(\mathbf{Q}_{1}^{-1}+\mathbf{F}_{2}^{T} \mathbf{Q}_{2}^{-1} \mathbf{F}_{2}\right)^{-1}\left(\left(\mathbf{F}_{2}^{T} \mathbf{Q}_{2}^{-1}\right)^{-1}\right)^{-1} \\
& =\left(\left(\mathbf{F}_{2}^{T} \mathbf{Q}_{2}^{-1}\right)^{-1}\left(\mathbf{Q}_{1}^{-1}+\mathbf{F}_{2}^{T} \mathbf{Q}_{2}^{-1} \mathbf{F}_{2}\right)\right)^{-1} \\
& =\left(\mathbf{Q}_{2} \mathbf{F}_{2}^{-T}\left(\mathbf{Q}_{1}^{-1}+\mathbf{F}_{2}^{T} \mathbf{Q}_{2}^{-1} \mathbf{F}_{2}\right)\right)^{-1} \\
& =\left(\mathbf{Q}_{2} \mathbf{F}_{2}^{-T} \mathbf{Q}_{1}^{-1}+\mathbf{F}_{2}\right)^{-1} \\
& =\left(\left(\mathbf{Q}_{2}+\mathbf{F}_{2} \mathbf{Q}_{1} \mathbf{F}_{2}^{T}\right) \mathbf{F}_{2}^{-T} \mathbf{Q}_{1}^{-1}\right)^{-1} \\
& =\mathbf{Q}_{1} \mathbf{F}_{2}^{T}\left(\mathbf{Q}_{2}+\mathbf{F}_{2} \mathbf{Q}_{1} \mathbf{F}_{2}^{T}\right)^{-1} \\
& =\mathbf{Q}_{1} \mathbf{F}_{2}^{T} \mathbf{Q}^{-1}=\mathbf{G} .
\end{aligned}
$$




\section{APPENDIX B}

\section{PROOF OF PROPOSITION 2}

This appendix presents the proof for Proposition 2. The derivation was previously published in [3].

Proof. The joint distribution of the states and the new observation is given by [1]

$$
\begin{aligned}
& p(\mathcal{X}, \mathbf{z} \mid \mathcal{Y}) \\
& =\mathcal{N}\left(\left[\begin{array}{c}
\mathcal{X} \\
\mathbf{z}
\end{array}\right] \mid\left[\begin{array}{c}
\hat{\mathcal{X}} \\
\overline{\mathbf{H}}_{\tau} \hat{\mathcal{X}}
\end{array}\right],\left[\begin{array}{cc}
\mathbf{P} & \mathbf{P} \overline{\mathbf{H}}_{\tau}^{T} \\
\overline{\mathbf{H}}_{\tau} \mathbf{P} & \overline{\mathbf{H}}_{\tau} \mathbf{P} \overline{\mathbf{H}}_{\tau}^{T}+\mathbf{R}
\end{array}\right]\right) \\
& =\mathcal{N}\left(\left[\begin{array}{c}
\mathcal{X} \\
\mathbf{z}
\end{array}\right] \mid\left[\begin{array}{c}
\hat{\mathcal{X}} \\
\mathbf{H} \hat{\mathbf{x}}_{\tau}
\end{array}\right],\left[\begin{array}{cc}
\mathbf{P} & \mathbf{P}_{\cdot \tau} \mathbf{H}^{T} \\
\mathbf{H} \mathbf{P}_{\tau} . & \mathbf{H} \mathbf{P}_{\tau} \mathbf{H}^{T}+\mathbf{R}
\end{array}\right]\right) .
\end{aligned}
$$

where $\overline{\mathbf{H}}_{\tau}$ is a sparse matrix defined such that $\overline{\mathbf{H}}_{\tau} \mathcal{X}=\mathbf{H x}_{\tau}$. The covariance notation used is $\mathbf{P}_{i, j}=\operatorname{Cov}\left(\mathbf{x}_{i}, \mathbf{x}_{j}\right)$ with the shorthand $\mathbf{P}_{i}=\mathbf{P}_{i, i}$ and $\mathbf{P}_{. i}=\operatorname{Cov}\left(\mathcal{X}, \mathbf{x}_{i}\right)$. Also, let $\mathbf{S}_{\tau}=$ $\mathbf{H P} \mathbf{P}_{\tau} \mathbf{H}^{T}+\mathbf{R}$ for simpler notation. Given an observation $\mathbf{z}$ at time $t_{\tau}$ the conditional distribution of $\mathcal{X}$ is given by [1]

$$
\begin{aligned}
& p(\mathcal{X} \mid \mathcal{Y}, \mathbf{z})= \mathcal{N}\left(\mathcal{X} \mid \hat{\mathcal{X}}+\mathbf{P}_{\cdot \tau} \mathbf{H}^{T} \mathbf{S}_{\tau}^{-1}\left(\mathbf{z}-\mathbf{H} \hat{\mathbf{x}}_{\tau}\right)\right. \\
&\left.\mathbf{P}-\mathbf{P}_{\cdot \tau} \mathbf{H}^{T} \mathbf{S}_{\tau}^{-1} \mathbf{H} \mathbf{P}_{\tau \cdot}\right) \\
&=\mathcal{N}\left(\mathcal{X} \mid \hat{\mathcal{X}}^{+}, \mathbf{P}^{+}\right)
\end{aligned}
$$

where the superscript + denotes the conditional quantity.

The full conditional distribution in (67) can be broken down into marginal distributions and cross-covariances for individual states as

$$
\begin{aligned}
\hat{\mathbf{x}}_{i}^{+} & =\hat{\mathbf{x}}_{i}+\mathbf{P}_{i, \tau} \mathbf{H}^{T} \mathbf{S}_{\tau}^{-1}\left(\mathbf{z}-\mathbf{H} \hat{\mathbf{x}}_{\tau}\right), \\
\mathbf{P}_{i, j}^{+} & =\mathbf{P}_{i, j}-\mathbf{P}_{i, \tau} \mathbf{H}^{T} \mathbf{S}_{\tau}^{-1} \mathbf{H} \mathbf{P}_{\tau, j}
\end{aligned}
$$

Letting $i=j=\tau$ in (68) directly gives (33).

The covariance matrix $\mathbf{P}$ is assumed to have a structure that satisfies

$$
\mathbf{P}_{i, j}=\mathbf{P}_{i, i+1} \mathbf{P}_{i+1}^{-1} \mathbf{P}_{i+1, j}, \quad i<j
$$

This assumption will be shown inductively to hold for the LGSSM, but can be shown to be a general result for inverses of symmetric block tridiagonal matrices using results in [4] under satisfied conditions. Through expansion of the assumption it follows that

$$
\mathbf{P}_{i, j}=\mathbf{P}_{i, i+1} \prod_{k=i+1}^{j-1} \mathbf{P}_{k}^{-1} \mathbf{P}_{k, k+1}=\mathbf{P}_{i, j-1} \mathbf{P}_{j-1}^{-1} \mathbf{P}_{j-1, j}
$$

and using the symmetry of the covariance matrix, $\mathbf{P}_{i, j}^{T}=\mathbf{P}_{j, i}$, similar results for $i>j$ are obtained by transposing (69) and (70).

The implication of the assumption (69) is that, given the covariance of each state and the cross-covariance of consecutive states, the cross-covariance between any states can be determined. The conditional distribution for the states $\mathbf{x}_{k}$ and the cross-covariances $\mathbf{P}_{k, k+1}$ between consecutive states for $k<\tau$ are derived from (68) as

$$
\begin{aligned}
\hat{\mathbf{x}}_{k}^{+} & =\hat{\mathbf{x}}_{k}+\mathbf{P}_{k, \tau} \mathbf{H}^{T} \mathbf{S}^{-1}\left(\mathbf{z}-\mathbf{H} \hat{\mathbf{x}}_{\tau}\right) \\
& =\hat{\mathbf{x}}_{k}+\mathbf{P}_{k, k+1} \mathbf{P}_{k+1}^{-1} \mathbf{P}_{k+1, \tau} \mathbf{H}^{T} \mathbf{S}^{-1}\left(\mathbf{z}-\mathbf{H} \hat{\mathbf{x}}_{\tau}\right) \\
& =\hat{\mathbf{x}}_{k}-\mathbf{P}_{k, k+1} \mathbf{P}_{k+1}^{-1}\left(\hat{\mathbf{x}}_{k+1}-\hat{\mathbf{x}}_{k+1}^{+}\right) \\
\mathbf{P}_{k, k+1}^{+} & =\mathbf{P}_{k, k+1}-\mathbf{P}_{k, \tau} \mathbf{H}^{T} \mathbf{S}^{-1} \mathbf{H} \mathbf{P}_{\tau, k+1} \\
& =\mathbf{P}_{k, k+1}-\mathbf{P}_{k, k+1} \mathbf{P}_{k+1}^{-1} \mathbf{P}_{k+1, \tau} \mathbf{H}^{T} \mathbf{S}^{-1} \mathbf{H} \mathbf{P}_{\tau, k+1} \\
& =\mathbf{P}_{k, k+1}-\mathbf{P}_{k, k+1} \mathbf{P}_{k+1}^{-1}\left(\mathbf{P}_{k+1}-\mathbf{P}_{k+1}^{+}\right) \\
& =\mathbf{P}_{k, k+1} \mathbf{P}_{k+1}^{-1} \mathbf{P}_{k+1}^{+},
\end{aligned}
$$

and

$$
\begin{aligned}
\mathbf{P}_{k}^{+}= & \mathbf{P}_{k}-\mathbf{P}_{k, \tau} \mathbf{H}^{T} \mathbf{S}^{-1} \mathbf{H} \mathbf{P}_{\tau, k} \\
= & \mathbf{P}_{k}-\mathbf{P}_{k, k+1} \mathbf{P}_{k+1}^{-1} \mathbf{P}_{k+1, \tau} \mathbf{H}^{T} \mathbf{S}^{-1} \mathbf{H} \\
& \mathbf{P}_{\tau, k+1} \mathbf{P}_{k+1}^{-1} \mathbf{P}_{k+1, k} \\
= & \mathbf{P}_{k}-\left(\mathbf{P}_{k, k+1}-\mathbf{P}_{k, k+1}^{+}\right) \mathbf{P}_{k+1}^{-1} \mathbf{P}_{k, k+1}^{T},
\end{aligned}
$$

using the assumption (69). By denoting $\mathbf{K}_{k}^{b}=$ $\mathbf{P}_{k, k+1} \mathbf{P}_{k+1}^{-1}$, (34) is obtained directly from (71). Similar derivations for $k>\tau$ results in (35), with $\mathbf{K}_{k}^{f}=\mathbf{P}_{k, k-1} \mathbf{P}_{k-1}^{-1}$.

Note that the update of the marginal posterior distribution only depends on the cross-covariances between consecutive states and not the full state covariance. As a result, only the state means, state covariances and cross-covariances between consecutive states are needed to compute the marginal posterior distribution.

The conditional cross-covariances in (68b) are for $j \leq \tau$ and $i<j$ obtained as

$$
\begin{aligned}
\mathbf{P}_{i, j}^{+} & =\mathbf{P}_{i, j}-\mathbf{P}_{i, \tau} \mathbf{H}^{T} \mathbf{S}_{\tau}^{-1} \mathbf{H} \mathbf{P}_{\tau, j}=\mathbf{P}_{i, i+1} \mathbf{P}_{i+1}^{-1} \mathbf{P}_{i+1, j} \\
& -\mathbf{P}_{i, i+1} \mathbf{P}_{i+1}^{-1} \mathbf{P}_{i+1, \tau} \mathbf{H}^{T} \mathbf{S}^{-1} \mathbf{H} \mathbf{P}_{\tau, j} \\
& =\mathbf{P}_{i, i+1} \mathbf{P}_{i+1}^{-1} \mathbf{P}_{i+1, j}+\mathbf{P}_{i, i+1} \mathbf{P}_{i+1}^{-1}\left(\mathbf{P}_{i+1, j}^{+}-\mathbf{P}_{i+1, j}\right) \\
& =\mathbf{P}_{i, i+1} \mathbf{P}_{i+1}^{-1} \mathbf{P}_{i+1, j}^{+}=\mathbf{P}_{i, i+1}^{+}\left(\mathbf{P}_{i+1}^{+}\right)^{-1} \mathbf{P}_{i+1, j}^{+}, \quad \text { (72) }
\end{aligned}
$$

using (71b) in the last equality, and for $j>\tau$ and $i<j$ as

$$
\begin{aligned}
\mathbf{P}_{i, j}^{+} & =\mathbf{P}_{i, j}-\mathbf{P}_{i, \tau} \mathbf{H}^{T} \mathbf{S}_{\tau}^{-1} \mathbf{H} \mathbf{P}_{\tau, j}=\mathbf{P}_{i, j-1} \mathbf{P}_{j-1}^{-1} \mathbf{P}_{j-1, j} \\
& -\mathbf{P}_{i, \tau} \mathbf{H}^{T} \mathbf{S}^{-1} \mathbf{H} \mathbf{P}_{\tau, j-1} \mathbf{P}_{j-1}^{-1} \mathbf{P}_{j-1, j} \\
& =\mathbf{P}_{i, j-1}^{+} \mathbf{P}_{j-1}^{-1} \mathbf{P}_{j-1, j}=\mathbf{P}_{i, j-1}^{+}\left(\mathbf{P}_{j-1}^{+}\right)^{-1} \mathbf{P}_{j-1, j}^{+}
\end{aligned}
$$

using (71b) to obtain the last equality, which are equivalent to (69) and (70), respectively, showing that the assumption (69) still holds for the conditional distribution.

Considering the discretized LGSSM in (4a) with the prior (4c) and no measurements, the covariance between states $\mathbf{x}_{i}$ and $\mathbf{x}_{j}$ for $i<j$ is given by

$$
\begin{aligned}
\mathbf{P}_{i, j} & =\operatorname{Cov}\left(\mathbf{x}_{i}, \mathbf{x}_{j}\right)=\mathrm{E}\left(\left(\mathbf{x}_{i}-\hat{\mathbf{x}}_{i}\right)\left(\mathbf{x}_{j}-\hat{\mathbf{x}}_{j}\right)^{T}\right) \\
& =\mathrm{E}\left(\left(\mathbf{x}_{i}-\hat{\mathbf{x}}_{i}\right)\left(\mathbf{F}_{j}\left(\mathbf{x}_{j-1}-\hat{\mathbf{x}}_{j-1}\right)+\mathbf{v}_{j}\right)^{T}\right) \\
& =\mathrm{E}\left(\left(\mathbf{x}_{i}-\hat{\mathbf{x}}_{i}\right)\left(\mathbf{x}_{j-1}-\hat{\mathbf{x}}_{j-1}\right)^{T}\right) \mathbf{F}_{j}^{T}=\mathbf{P}_{i, j-1} \mathbf{F}_{j}^{T} \\
& =\mathbf{P}_{i, j-1} \mathbf{P}_{j-1}^{-1} \mathbf{P}_{j-1} \mathbf{F}_{j}^{T}=\mathbf{P}_{i, j-1} \mathbf{P}_{j-1}^{-1} \mathbf{P}_{j-1, j} .
\end{aligned}
$$

This shows inductively that the assumption (69) holds initially before conditioning on measurements, concluding the proof. 


\section{REFERENCES}

[1] F. Gustafsson, Statistical Sensor Fusion, 2nd ed. Lund, Sweden: Studentlitteratur, 2012.

[2] R. A. Horn and C. R. Johnson, Matrix Analysis, 2nd ed. New York, NY, USA: Cambridge University Press, 2013.

[3] C. Veibäck, "Tracking of animals using airborne cameras," Licentiate's Thesis, Linköping University, Nov. 2016.

[4] G. Meurant, "A review on the inverse of symmetric tridiagonal and block tridiagonal matrices," SIAM Journal on Matrix Analysis and Applications, vol. 13, no. 3, pp. 707-728, 1992. 